\title{
Stochastic Generation of Explicit Pore Structures By Thresholding
} Gaussian Random Fields

\author{
Jeffrey D. Hyman ${ }^{\mathrm{a}}$, C. Larrabee Winter ${ }^{\mathrm{b}}$ \\ a Program in Applied Mathematics, University of Arizona, Tucson, AZ 85721-0089, USA \\ Computational Earth Science, Earth and Environmental Sciences, (EES - 16) and Center for Nonlinear Studies, Los \\ Alamos Nat'l Lab., Los Alamos, NM 87544, USA \\ ${ }^{b}$ Department of Hydrology and Water Resources, Program in Applied Mathematics, University of Arizona, Tucson, AZ \\ 85721-0011, USA
}

\section{Abstract}

We provide a description and computational investigation of an efficient method to stochastically generate realistic pore structures. Smolarkiewicz and Winter introduced this specific method in Pores resolving simulation of Darcy flows (J. Comput. Phys. 229 (9) (2010) 3121-3133) without giving a complete formal description or analysis of the method, or indicating how to control the parameterization of the ensemble. We address both issues in this paper. The method consists of two steps. First, a realization of a correlated Gaussian field, or topography, is produced by convolving a prescribed kernel with an initial field of independent, identically distributed random variables. The intrinsic length scales of the kernel determines the correlation structure of the topography. Next, a sample pore space is generated by applying a level threshold to the Gaussian field realization: points are assigned to the void phase or the solid phase depending on whether the topography over them is above or below the threshold. Hence, the topology and geometry of the pore space depend on the form of the kernel and the level threshold. Manipulating these two user prescribed quantities allows good control of pore space observables, in particular the Minkowski functionals. Extensions of the method to generate media with multiple pore structures and preferential flow directions are also discussed. To demonstrate it's usefulness, the method is used to generate a pore space with physical and hydrological properties similar to a sample of Berea sandstone.

Keywords: porous media, stochastic methods, Minkowski functionals, Direct numerical simulation

\section{Introduction}

Computational experiments are an important tool for studying the flow of fluids through porous media. This is especially true of small scale flow systems where porous media are represented as microstructures composed of interconnected channels (pores) embedded in an otherwise impermeable solid where physical experiments cannot usually show much detail. Porous microstructures, pore spaces for short, are characterized by their topology, primarily their connectivity, and their observable geometry including pore radii, pore lengths, and pore wall surface area; and spatial statistics, especially correlation structure. Direct numerical simulations of flow through pore spaces yield insights into the effects of pore space characteristics on microscopic flow properties such as streamline tortuosity $[2,3]$ and macroscopic flow properties such as permeability $[4,5]$. The spatially variable resistance offered by the solid phase results in non-uniform fluid velocity fields where the observed dynamics range from stagnation to the chaotic separation of fluid particle trajectories [6]. Modeling these phenomena has applications that vary from groundwater flow and transport [7, 8], to geophysical applications such as petroleum extraction [4] and carbon sequestration [9], to filter and textile design [10], the delivery of drugs to tumors [11], and improved baking technology [12].

Email addresses: jhyman@lanl.gov (Jeffrey D. Hyman), winter@email.arizona.edu (C. Larrabee Winter) 
Detailed numerical experiments require both a fluid dynamics simulator capable of resolving flow on the relatively small scales of explicit porous microstructures and a method for obtaining pore spaces with realistic characteristics at desired scales. We focus on the second requirement in this paper by analyzing and extending a stochastic generation method that produces realistic realizations of two or three dimensional pore spaces by thresholding Gaussian random fields. Smolarkiewz and Winter introduced the specific method in [1] without giving a complete formal description or analysis of the method, or indicating how to control the parameterization of the ensemble. Quiblier [13] introduced a similar method, which was used by Adler et al. [14] and Yao et al. [15], that reproduces a desired correlation structure of a pore sample. However, neither group indicated how to control the physical properties of the ensemble by manipulating parameters of the generation process, which is the issue we address in this paper. The generation process yields realizations with specified values of the first three Minkowski functionals of integral geometry: pore volume, porosity, specific surface area, and mean curvature $[16,17,18]$. A theorem of Hadwiger assures that all continuous rigid motion invariant valuations on convex bodies in three dimensions are spanned by the first four Minkowski functionals [19]. Therefore, a wide class of porous microstructures can be generated using this procedure.

The method is motivated by the desire to sample realizations from pore space ensembles whose geometric observables, e.g., the Minkowski functionals, correspond to those of sample images of real pore spaces. High resolution images of porous microstructures can be obtained by applying X-ray tomography, or related techniques, to physical samples of real porous media [20,21, 22]. The availability of samples and the costs of imaging can limit the number of realizations that can be taken directly from images [23]. Random sampling from an ensemble of pore spaces defined by representative statistics from actual images is a cost-effective means of producing large numbers of realizations that are similar to imaged pore spaces. The statistics can be chosen to reproduce correlation structures. For example, Okabe and Blunt [24] used multiple point statistics based on two-dimensional cross sections to generate three-dimensional pore spaces, Adler et al. [14] use explicit correlation functions to generate correlated pore spaces based on Gaussian fields, and Manwart et al. [25] reconstructs samples based on pore size distribution functions, linear path functions, and two-point probabilities using a simulated annealing algorithm. Alternatively, values of physical observables taken from images, e.g., the Minkowski functionals [16, 17, 18], have been used to create realizations corresponding to real porous media $[26,27]$. Arns et al. [28] compare the abilities of pore space simulations based on overlapping spheres, leveled-waves, and Voronoi tesselations to reproduce Minkowski functionals of a sandstone. Methods that randomly place geometrical objects in a domain until a prescribed pore space attribute, e.g., porosity, is obtained have also been used. Matyka et al. [2] generate a pore space realization with given porosity by randomly placing squares into a two dimensional domain; Duda et al. [29] extended this method to three dimensions using freely overlapping cubes. A similar method uses random packings of equal and unequal sized spheres and circles which are not allowed to overlap [30]. Such methods are inherently sequential.

The method we investigate in this paper is based on generating Gaussian random fields and thresholding them so as to produce statistics corresponding to specified observable characteristics [1]. The method, which is highly parallel, consists of two steps. First, a realization of a correlated Gaussian field, or topography, is produced by convolving a prescribed kernel with an initial field of independent, identically distributed random variables. The intrinsic length scales of the kernel $\lambda$ determine the correlation structure of the topography. Next, a sample pore space is generated by applying a level threshold $\gamma$ to the Gaussian field realization: points are assigned to the void phase or the solid phase depending on whether the surface over them is above or below $\gamma$. Hence, the topology and geometry of the pore space depend on the form of the kernel and the parameters $\lambda$ and $\gamma$. We concentrate on the properties of pore spaces generated with Gaussian kernels. We call $(\lambda, \gamma)$ a generation pair. Manipulation of the generation pair allows good control of pore space observables, and we develop look-up tables and figures that map generation pairs onto specific values of the Minkowski functionals.

The mathematical literature on Gaussian fields has mostly concentrated on percolation of connected paths through thresholded fields $[31,32]$ or on the evolution of Gaussian fields under Gibbsian dynamics [33]. Thresholded Gaussian fields have also been used to generate pore spaces with specific correlation structures $[13,14,15]$. In those applications the kernel is selected to reproduce the desired correlation structure, and observable properties like values of the Minkowski functionals are secondary. Since the primary object of the method we describe is to generate pore spaces with specific observable properties, 
kernels can be chosen for convenience and the correlation structure of the pore space is a consequence of the generation pair.

We discuss the basic simulation technique for generating isotropic stationary pore spaces in Section 2. We characterize the probability ensembles from which stationary simulations are drawn and demonstrate how the correlation structures of the Gaussian topographies depends on $\lambda$. By defining pore space membership using an indicator function, $\chi(\mathbf{x})$, the process is amenable to analysis via standard probability theory, and we relate $\lambda$ and $\gamma$ to $\mathbb{P}[\chi(\mathbf{x})=1, \chi(\mathbf{y})=1]$, the joint probability (and spatial correlation) of pore space membership. The practical import of these calculations is to allow correlations to be calculated from a generation pair once the pair has been chosen to produce desired values of the observables. Alternatively, a generation pair can be chosen to produce a specific correlation structure in which case the observables would follow from the correlation structure in a fashion similar to $[13,14,15]$. However, that is not our focus. In Section 3 we simulate a range of pore spaces by relating $\lambda$ and $\gamma$ to the first three Minkowski functionals. We illustrate the flexibility of the method by constructing anisotropic pore spaces and also a particular class of non-stationary porous media in Section 4. In Section 5, we simulate a sample of Berea sandstone by generating a pore space with similar Minkowski functionals and bulk hydrological properties. We conclude with a brief summary of the method and a few remarks in Section 6 .

\section{Stochastic Generation Method}

In this paper, a stochastic porous medium is an ensemble of pore space realizations $\mathbb{S} \subset \mathbb{R}^{n}$ where usually $n=3$. Each $\mathbb{S}=\mathbb{P} \cup \mathbb{M}$ consists of a network of interconnected pores, $\mathbb{P}$, embedded in a solid matrix, $\mathbb{M}$. The internal boundary between the pores and the solid matrix is denoted by $\partial \mathbb{P}=\partial \mathbb{M}=\mathbb{P} \cap \mathbb{M}$. The volume of $\mathbb{S}$ is the sum of the volumes of the pore space and solid matrix, $\mathbb{V}_{\mathbb{S}}=\mathbb{V}_{\mathbb{P}}+\mathbb{V}_{\mathbb{M}}$. Level-set percolation $[34,35]$ is used to generate realizations of porous media from underlying random topographies. Each pore space realization is derived by applying a fixed level threshold, $\gamma$, to an associated hypersurface, or random topography field, $T(\mathbf{x})$. A realization of $T$ is derived by convolving a fixed kernel, $k(\mathbf{x})$, with a realization, $u(\mathbf{x})$, of a random field, $U$, whose elements are independent, identically distributed uniform random variables, i.e.,

$$
T(\mathbf{x})=\int_{\mathbb{R}^{n}} k(\mathbf{x}-\mathbf{y}) u(\mathbf{y}) d \mathbf{y} .
$$

\subsection{Generation Procedure}

The generation method is summarized by the pseudo-code:

I Populate $\mathbb{S} \subset \mathbb{R}^{n}$ with a realization, $u(\mathbf{x})$, of a random field whose elements are independent, identically distributed random variables sampled from the continuous uniform distribution on the interval $[a, b]$. Here, $[a, b]$ generally equals $[0,1]$.

II Generate a correlated random topography, $T=k * u$, by convolving $u$ with a deterministic kernel, $k$. It is convenient if $\int_{\mathbb{R}^{n}} k(\mathbf{x}) d \mathbf{x}=1$.

III Produce a pore-space realization, $\mathbb{P}$, by thresholding given $T(\mathbf{x})$ with a level threshold $\gamma \in$ $[a, b]: \mathbb{P}=\{\mathbf{x} \in \mathbb{S} \mid T(\mathbf{x})<\gamma\}$. The resulting pore space is equivalent to the indicator function

$$
\chi(\mathbf{x})= \begin{cases}1, & \text { if } T(\mathbf{x})<\gamma \\ 0, & \text { if } T(\mathbf{x}) \geq \gamma\end{cases}
$$

To provide physical intuition, Fig. 1 displays images of a two-dimensional version of the method. The left subfigure, Fig. 1a, is the correlated topography field $T$; the middle subfigure, Fig. 1b, shows the threshold level applied to $T$; and the resulting two-dimensional pore space, $\mathbb{P}$, is the right subfigure, Fig. 1c. The choice of the convolution kernel $k$ and the level threshold $\gamma$ dictate the geometric and topological features of the pore space. As $\gamma$ rises, the volume of the void space, $\mathbb{V}_{\mathbb{P}}$, increases and thus the medium's porosity, $\mathbb{V}_{\mathbb{P}} / \mathbb{V}_{\mathbb{S}}$, increases. The subfigures also illustrate how the correlation structure of $T$ is transferred into $\mathbb{P}$. A 
topography with a short correlation length will have narrow peaks and valleys that become small pores once $\gamma$ is applied. Wider pores are created using topographies with longer correlation lengths. The decay and support of $k$ determine the covariance matrix associated with $T$, and the smoothness of $T$ depends upon the smoothness of $k$.

(a) $T(\mathbf{x})$



(b) $\mathbb{P}=\{\mathbf{x} \in \mathbb{S} \mid T(\mathbf{x})<\gamma\}$

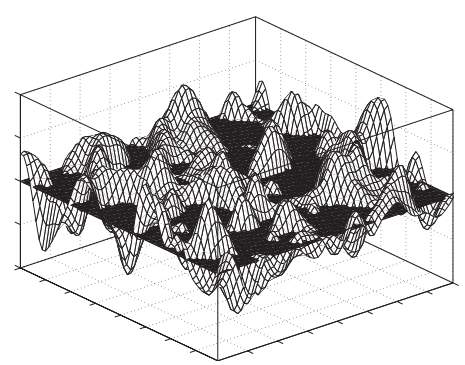

(c) $\chi(\mathbf{x})$

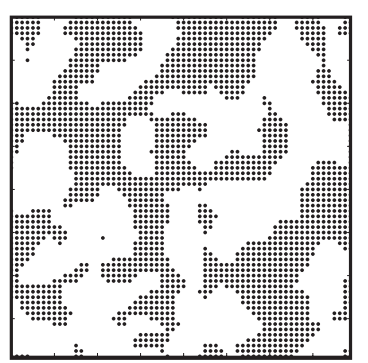

Fig. 1: An example of how the method generates a pore space realization. The left subfigure is the random correlated topography $T$, the middle subfigure shows the threshold level $\gamma$ applied to the topography in the left subfigure, and the right subfigure is the resulting two-dimensional pore space. The solid matrix is shown in black.

Remarks: (i) If the kernel $k$ is symmetric, then the topography $T$ is isotropic because the initial field $U$ is also isotropic. (ii) $T$ is stationary because $U$ is stationary. (iii) $\mathbb{E}[T(\mathbf{x})]=\mu_{T}=\mu_{U}$ from the definitions of the $U$ and $T$ fields, where $\mu_{T}$ and $\mu_{U}$ denote the means of $T$ and $U$. (iv) The topography $T$ is asymptotically normal, $T(\mathbf{x}) \sim N\left(\mu_{T}, \sigma_{T}^{2}\right)$, with mean $\mu_{T}$ and variance $\sigma_{T}^{2}$. This property follows from the version of the Central Limit Theorem due to Liapunov [36]. (v) A generalization of a theorem by Lindeberg and Levy to the multi-dimensional Central Limit Theorem [36] implies that $T(\mathbf{x})$ and $T(\mathbf{y})$ are joint normally distributed, i.e., $T(\mathbf{x}), T(\mathbf{y}) \sim N\left(\boldsymbol{\mu}_{T}, \boldsymbol{\Sigma}_{T}\right)$ for all $\mathbf{x}, \mathbf{y}$; where $\boldsymbol{\mu}_{T}=\left(\mu_{T}, \mu_{T}\right)$ and

$$
\boldsymbol{\Sigma}_{T}(\mathbf{x}, \mathbf{y})=\left[\begin{array}{cc}
\sigma_{T}^{2} & C_{T}(\mathbf{x}, \mathbf{y}) \\
C_{T}(\mathbf{x}, \mathbf{y}) & \sigma_{T}^{2}
\end{array}\right],
$$

where $C_{T}(\mathbf{x}, \mathbf{y})=\operatorname{Cov}[T(\mathbf{x}), T(\mathbf{y})]$ denotes the covariance between $T(\mathbf{x})$ and $T(\mathbf{y})$ located at points $\mathbf{x}, \mathbf{y} \in \mathbb{S}$. The topography $T$ can be rendered anisotropic by choosing an asymmetric kernel $k$, while properties (ii)-(v) are unaffected by the anisotropy of $T$.

\subsection{Kernel Examples}

To provide concrete results, especially about the covariance $C_{T}(\mathbf{x}, \mathbf{y})$, we discuss three different options for the convolution kernel: (i) uniform weighting over a finite rectilinear domain, (ii) symmetric Gaussian kernels that weigh contributions to the topography by distance from the center point, decaying exponentially with distance, and (iii) a symmetric kernel produced by iterative sweeps of a low-pass filter. All three examples yield topographies that are normally distributed per our earlier Remarks (iv) and (v). Although we only provide three examples, the generation method is general enough that any numerical convolution with a desired kernel can be used to derive $T$. The method is also general enough to be applied in arbitrary dimensions, but we focus on the three dimensional case because our goal is generating realistic porous microstructures.

In the following, $u$ is a realization of the random field $U$ defined on a finite three dimensional Cartesian grid with equal spacing $(\delta x=\delta y=\delta z)$ between grid nodes $(i, j, k)$. Volumes are rectilinear with nodes labeled by $i=0,1, \ldots, \sqrt[3]{N} ; j=0,1, \ldots, \sqrt[3]{N} ;$ and $k=0,1, \ldots, \sqrt[3]{N}$, where $\sqrt[3]{N}$ is the number of grid nodes in each direction and $N$ the total number of grid nodes. The location $(i \delta x, j \delta y, k \delta z)$ on the grid is denoted by $\mathbf{x}_{i, j, k}$, $u\left(\mathbf{x}_{i, j, k}\right)$ is $u_{i, j, k}$, and $\mathbb{V}_{i, j, k}$, is the volume centered on $x_{i, j, k}$. Again we use $C_{T}(\mathbf{x}, \mathbf{y})=\operatorname{Cov}[T(\mathbf{x}), T(\mathbf{y})]$ to denote the covariance between $T(\mathbf{x})$ and $T(\mathbf{y})$ located at points $\mathbf{x}, \mathbf{y} \in \mathbb{S}$. 


\subsubsection{Uniform Kernel}

The $n$ dimensional uniform kernel generates $T(\mathbf{x})$ as the average of $u(\mathbf{x})$ at all points in an $n$ dimensional rectilinear volume $\mathbb{V}$, centered at $\mathbf{x}$

$$
T(\mathbf{x})=\frac{1}{\mathbb{V}(\mathbf{x})} \int_{\mathbb{R}^{n}} u(\mathbf{y}) \chi_{\mathbb{V}(\mathbf{x}-\mathbf{y})}(\mathbf{x}-\mathbf{y}) d \mathbf{y},
$$

where $\chi_{\mathbb{V}(\mathbf{x})}(\mathbf{x})$ is the indicator function on $\mathbb{V}(\mathbf{x})$. The associated length scales of this kernel, $\lambda$, are equal to the lengths of the sides of the $n$ dimensional rectilinear volume. On the computational grid, this corresponds to $\lambda_{i}=2 a_{i}+1$ for $i=1, \ldots, n$ in each of the principal directions where $a_{i}$ depends on the length of the rectilinear volume in the $i$ th direction. Explicitly, in three dimensions this convolution is

$$
T\left(\mathbf{x}_{i, j, k}\right)=\left[\left(2 a_{x}+1\right)\left(2 a_{y}+1\right)\left(2 a_{z}+1\right)\right]^{-1} \sum_{n=i-a_{x}}^{i+a_{x}} \sum_{m=j-a_{y}}^{j+a_{y}} \sum_{l=k-a_{z}}^{k+a_{z}} u_{n, m, l} .
$$

Anisotropy may be introduced into the topography by varying the length scales $\lambda_{i}$ in desired directions. The number of floating point operations (FLOPs) required by (5) is $\mathcal{O}(m * N)$, where $N$ is the number of points in the computational grid, and $m$ is the number of points in the $n$ dimensional rectilinear volume $\mathbb{V}$.

The covariance between two points, $T(\mathbf{x})$ and $T(\mathbf{y})$, depends on the number of points common to $\mathbb{V}(\mathbf{x})$ and $\mathbb{V}(\mathbf{y})$. A straightforward calculation shows that

$$
C_{T}(\mathbf{x}, \mathbf{y})=\sigma_{U}^{2} \Delta(\mathbf{x}, \mathbf{y}) /\left(\Pi_{i=1}^{n} \lambda_{i}\right)^{2} .
$$

where $\Delta(\mathbf{x}, \mathbf{y})$ is the number of grid nodes in $\mathbb{V}(\mathbf{x}) \cap \mathbb{V}(\mathbf{y})$ and $\sigma_{U}^{2}$ is the variance of the random field $U$. It follows from (6), that $C_{T}(\mathbf{x}, \mathbf{y})=0$ when the distance between $\mathbf{x}$ and $\mathbf{y}$ is sufficiently large such that $\mathbb{V}(\mathbf{x}) \cap \mathbb{V}(\mathbf{y})=\emptyset$.

\subsubsection{Gaussian Function}

In $n$ dimensions the Gaussian kernel is

$$
k(\mathbf{x})=\frac{1}{(2 \pi)^{n / 2} \sqrt{\operatorname{Det}(\boldsymbol{\Lambda})}} \exp \left[-\mathbf{x}^{\prime} \boldsymbol{\Lambda}^{-1} \mathbf{x} / 2\right],
$$

where $\boldsymbol{\Lambda}$ is symmetric matrix of length scales whose elements $\lambda_{i}$ determine the spread of $k(\mathbf{x})$ in various directions. Gaussian kernels were used to generate pore space realizations in $[3,5,6,37]$. Isotropic topographies can be created by defining $\boldsymbol{\Lambda}$ as a diagonal matrix and assigning the same length scale $\lambda$ to every direction. See Section 4.1 for examples of anisotropic topographies generated with a Gaussian kernel.

The convolution theorem allows for an efficient evaluation of the convolution even though the Gaussian kernel (7) has infinite support. The convolution is achieved by transforming a realization of the random field into Fourier space using the discrete Fourier transform, $\mathcal{F}[\cdot]$, point-wise multiplication by the discrete Fourier transform of (7), and then applying the inverse transform to the product, i.e., $T(\mathbf{x})=$ $\mathcal{F}^{-1}[\mathcal{F}[k(\mathbf{x})] \odot \mathcal{F}[u(\mathbf{x})]]$, where $k(\mathbf{x})$ is defined in $(7)$ and $\odot$ denotes point wise multiplication. If the discretization of the domain is designed so the fast Fourier transform can be used, the number of FLOPs required is $\mathcal{O}(N \log N)$.

Since all topographies in the provided examples are stationary random fields with $\mu_{T}=\mu_{U}$, Remarks (ii) and (iii), the covariance of any topography at arbitrary points $\mathbf{x}, \mathbf{y} \in \mathbb{S} \subset \mathbb{R}^{n}$ is $C_{T}(\mathbf{x}, \mathbf{y})=\mathbb{E}[(T(\mathbf{x})-$ $\left.\left.\mu_{U}\right)\left(T(\mathbf{y})-\mu_{U}\right)\right]$. Using the definition of $T=k * u$ and elementary properties of expectation,

$$
\begin{aligned}
\mathbb{E}\left[\left(T(\mathbf{x})-\mu_{U}\right)\left(T(\mathbf{y})-\mu_{U}\right)\right] & =\mathbb{E}\left[\int_{\mathbb{R}^{n}}\left[u(\boldsymbol{\xi})-\mu_{U}\right] k(\mathbf{x}-\boldsymbol{\xi}) d \boldsymbol{\xi} \int_{\mathbb{R}^{n}}\left[u(\boldsymbol{\psi})-\mu_{U}\right] k(\mathbf{y}-\boldsymbol{\psi}) d \boldsymbol{\psi}\right] \\
& =\mathbb{E}\left[\int_{\mathbb{R}^{n}} \int_{\mathbb{R}^{n}} k(\mathbf{x}-\boldsymbol{\xi}) k(\mathbf{y}-\boldsymbol{\psi})\left[u(\boldsymbol{\xi})-\mu_{U}\right]\left[u(\boldsymbol{\psi})-\mu_{U}\right] d \boldsymbol{\xi} d \boldsymbol{\psi}\right] \\
& =\int_{\mathbb{R}^{n}} \int_{\mathbb{R}^{n}} k(\mathbf{x}-\boldsymbol{\xi}) k(\mathbf{y}-\boldsymbol{\psi}) \mathbb{E}\left[\left(u(\boldsymbol{\xi})-\mu_{U}\right)\left(u(\boldsymbol{\psi})-\mu_{U}\right)\right] d \boldsymbol{\xi} d \boldsymbol{\psi}
\end{aligned}
$$


The limits of integration in (8) apply because the spatial extent of the domain is much greater than $\lambda_{1}, \ldots, \lambda_{n}$ the length scales of $k$. When $\boldsymbol{\xi} \neq \boldsymbol{\psi}$, the random variables $u(\boldsymbol{\xi})$ and $u(\boldsymbol{\psi})$ are independent and $\mathbb{E}\left[\left(u(\boldsymbol{\xi})-\mu_{U}\right)\left(u(\boldsymbol{\psi})-\mu_{U}\right)\right]=0$. Otherwise $\mathbb{E}\left[\left(u(\boldsymbol{\xi})-\mu_{U}\right)\left(u(\boldsymbol{\psi})-\mu_{U}\right)\right]=\sigma_{U}^{2}$. Hence,

$$
C_{T}(\mathbf{x}, \mathbf{y})=\sigma_{U}^{2} \iint_{\boldsymbol{\xi}=\boldsymbol{\psi}} k(\mathbf{x}-\boldsymbol{\xi}) k(\mathbf{y}-\boldsymbol{\psi}) d \boldsymbol{\xi} d \boldsymbol{\psi},
$$

where $\sigma_{U}^{2}$ is the variance of the random field $U$.This integral can be taken along $\mathbf{t}=\left(t_{1}, \ldots, t_{n}\right)$ the line in $\mathbb{R}^{n}$ where $\boldsymbol{\xi}=\boldsymbol{\psi}$, leading to

$$
C_{T}(\mathbf{x}, \mathbf{y})=\frac{\sigma_{U}^{2}}{\left(2 \pi^{1 / 2}\right)^{n}\left(n \Pi_{i=1}^{n} \lambda_{i}\right)^{2}} \exp \left[-\frac{1}{4}\left(\sum_{i=1}^{n} \frac{\left(x_{i}-y_{i}\right)^{2}}{\lambda_{i}}\right)\right] .
$$

In particular, $\operatorname{Var}[T(\mathbf{x})]=\sigma_{U}^{2} /(2 \pi)^{n / 2}\left(n \prod_{i=1}^{n} \lambda_{i}\right)^{2}$. Furthermore, $C_{T}(\mathbf{x}, \mathbf{y}) \rightarrow 0$ as $\|\mathbf{x}-\mathbf{y}\| \rightarrow \infty$.

\subsubsection{Low-pass Filtering}

Smolarkiewicz and Winter [1] perform the convolution using a series of applications of a tensor product. Due to the difficulties of analyzing the correlation structure of $T$ that it creates, it is not considered later in this paper. Briefly, a realization $u$ of the $U$ random field, is low-pass filtered using some $m$ consecutive applications of the tensor product $u^{u l t}=u^{u l t x} \otimes u^{u l t y} \otimes u^{u l t z}$. Each alternate-direction application of the one-dimensional filter $u^{\text {ultx }}, u^{\text {ulty }}$ or $u^{\text {ultz }}$ employs the trapezoidal rule integral over two adjacent grid boxes - e.g., $u_{\mathrm{i}}^{\text {ultx }}=0.25\left(u_{\mathrm{i}+1, \mathrm{j}, \mathrm{k}}+2 u_{\mathrm{i}, \mathrm{j}, \mathrm{k}}+u_{\mathrm{i}-1, \mathrm{j}, \mathrm{k}}\right)$ in the direction of $x$, with the amplitude response $\hat{u}^{u l t x}(k)=\hat{u}(k) 0.5(1+\cos (k \Delta x))$, where $k$ is the wave-number of the Fourier mode $\hat{u}(k) e^{i k x}$ on the grid. The associated length scale is symmetric and equal to $\lambda=2 m+1$. Thus, a higher number of applications of the low pass filter is equivalent to using a wider kernel in the convolution. Anisotropy can be introduced by using an unequal number of sweeps in different directions. This method requires $\mathcal{O}(N)$ FLOPs.

\subsection{Distribution of the Indicator Function}

Basic facts about the indicator function $\chi(\mathbf{x}), \chi(\mathbf{x})=1$ void and $\chi(\mathbf{x})=0$ solid, that defines the pore space include (i) the probability that a point is in the pore space is given by $\mathbb{P}[\chi(\mathbf{x})=1]=\mathbb{P}[T(\mathbf{x})<\gamma]$ for all $\mathbf{x}$, (ii) the two-point probability is given by $\mathbb{P}[\chi(\mathbf{x})=1, \chi(\mathbf{y})=1]=\mathbb{P}[T(\mathbf{x})<\gamma, T(\mathbf{y})<\gamma]$ for all $\mathbf{x}$ and $\mathbf{y}$ (similar definitions apply to $\mathbb{P}[\chi(\mathbf{x})=1, \chi(\mathbf{y})=0], \mathbb{P}[\chi(\mathbf{x})=0, \chi(\mathbf{y})=1]$, and $\mathbb{P}[\chi(\mathbf{x})=0, \chi(\mathbf{y})=0]$ ) and (iii) each $\chi(\mathbf{x})$ is a Bernoulli distributed random variable, with mean $\mu_{\chi}=p$ and variance $\sigma_{\chi}^{2}=p(1-p)$ where

$$
p=\mathbb{P}[\chi(\mathbf{x})=1]=\frac{1}{2}+\frac{1}{2} \operatorname{Erf}\left[\frac{\sqrt{\lambda}\left(\gamma-\mu_{U}\right) \pi^{1 / 4}}{\sigma_{U} \sqrt{2}}\right] .
$$

Equation (11) provides a closed form expression for the expected porosity, $\mathbb{E}\left[\mathbb{V}_{P} / \mathbb{V}_{\mathbb{S}}\right]$ void volume over total volume, of a pore space. The form of this equation results from the normality of the topography $T$, which is a result of the central limit theorem not the kernel choice. The particular coefficients vary with kernel choice. For a fixed kernel width, the expected porosity rises nonlinearly with the level threshold $\gamma$. For any given value of $\gamma$, the expected porosity also depends upon the kernel's associated length scale, $\lambda$. However, as can be seen in (6) and (10), as $\lambda$ increases, the variance of the topography $T$ decreases. For sufficiently large values of $\lambda$, (11) degenerates into a heavy-side function centered about $\mu_{U}$. To stop this from occurring, the topography can be mapped so that $\min (T)=0$ and $\max (T)=1$, prior to the application of the level threshold $\gamma$. This mapping preserves the correlation structure induced by the kernel and renders the result of applying $\gamma$ the same regardless of the associated length scale. Although multiple pairs $(\lambda, \gamma)$ can yield the same value pdf for $\chi(\mathbf{x})$, this is an aftereffect of the method since $\lambda$ and $\gamma$ are chosen independently to produce a particular pore space and not vice versa, i.e., pore spaces are not derived ex post facto from $\lambda$ and $\gamma$. Additionally, the joint influence of $\lambda$ and $\gamma$ on the 2nd-order pdf is more complex and is provided below (12). 
Because $\mathbb{P}[\chi(\mathbf{x})=1, \chi(\mathbf{y})=1]=\mathbb{P}[T(\mathbf{x})<\gamma, T(\mathbf{y})<\gamma]$, it follows from the Central Limit Theorem that

$$
\mathbb{P}[\chi(\mathbf{x})=1, \chi(\mathbf{y})=1]=\frac{1}{2 \pi|\boldsymbol{\Sigma}|} \int_{-\infty}^{\gamma} \int_{-\infty}^{\gamma} \exp \left[-\frac{1}{2}\left(\mathbf{T}-\boldsymbol{\mu}_{T}\right)^{\prime} \boldsymbol{\Sigma}_{T}^{-1}\left(\mathbf{T}-\boldsymbol{\mu}_{T}\right)\right] d T_{x} d T_{y},
$$

as $\lambda$ increases. Here $\boldsymbol{\mu}_{T}$ and $\boldsymbol{\Sigma}_{T}$ are defined in $(3)$, and $\mathbf{T}=\left(T_{x}, T_{y}\right)$. Expressions for $\mathbb{P}[\chi(\mathbf{x})=0, \chi(\mathbf{y})=$ $1], \mathbb{P}[\chi(\mathbf{x})=1, \chi(\mathbf{y})=0]$, and $\mathbb{P}[\chi(\mathbf{x})=1, \chi(\mathbf{y})=1]$ can be derived similarly. Combining (11) and (12) provides the covariance of the indicator function

$$
\begin{aligned}
\operatorname{Cov}[\chi(\mathbf{x}), \chi(\mathbf{y})] & =\mathbb{E}[\chi(\mathbf{x}), \chi(\mathbf{y})]-\mu_{\chi}^{2} \\
& =1 \times 1 \times \mathbb{P}[\chi(\mathbf{x})=1, \chi(\mathbf{y})=1]-\mathbb{P}[\chi(\mathbf{x})=1]^{2} \\
& =\mathbb{P}[\chi(\mathbf{x})=1, \chi(\mathbf{y})=1]-\mathbb{P}[\chi(\mathbf{x})=1]^{2} .
\end{aligned}
$$

This analysis applies to any number of points $\mathbf{x}, \mathbf{y}, \mathbf{z}, \ldots$ so the joint distribution for any number of elevations has the same form as (12) except the dimensions are larger.

Adler et al. [14] arrived at a similar expression to (12) when considering the method of Quiblier [13] to generate random porous media. In that method a field of uncorrelated random Gaussian variables is first passed through a linear filter, a discrete convolution, and then a nonlinear filter, similar to the threshold level (2), which yields a realization that reproduces the autocorrelation function of a sample of real media. They expand the integral using Hermite polynomials and obtain an infinite summation that defines the correlation function of the pore space in terms of the correlation function of the correlated field of Gaussian random variables. The higher order moments of their generated pore spaces are discussed in [15].

\section{Pore-Space Characterization}

The physical characteristics of each porous microstructure are determined by the length-scale of the chosen convolution kernel, $\lambda$, and the selected threshold level, $\gamma$. The support, symmetry, and decay rate of the kernel determine the correlation structure of the topography, and the level threshold determines the resulting pore space. These two user-prescribed quantities interact non-linearly to generate the geometry and topology of the void space, and they can be chosen to create porous microstructures with desired physical characteristics. Hereafter we refer to the kernel length scale $\lambda$ and level threshold $\gamma$, as a generation pair, even though $\lambda$ may be a matrix.

In this section, we provide a computational investigation of how the choice of generation pair affects geometric observables of a pore space realization, specifically the first three Minkowski functionals for a porous medium $[17,18,38,16]$, and pore network topology. The topographies in this study are generated by convolving the symmetric Gaussian kernel with independent realizations of the initial random field. This kernel is selected because of the smoothness it induces in the topography and the computational efficiency achieved by implementing the fast Fourier transform, cf. Section 2.2.2. The study was also performed using the uniform kernel, Section 2.2.1, but the results are similar to those reported below and are therefore omitted. The initial field of random variables is drawn from the continuous uniform distribution on the interval $[0,1]$ (mean $\mu_{U}=1 / 2$ and variance $\sigma_{U}^{2}=1 / 12$ ). Each pore-space has dimensions $L_{x}=L_{y}=L_{z}=$ $1.27 \cdot 10^{-2} \mathrm{~m}$, with volume $\mathbb{V}=2.05 \cdot 10^{-6} \mathrm{~m}^{3}$. The domain is discretized using a uniform Cartesian grid with a grid spacing of $\delta x=10^{-4} \mathrm{~m}$. This domain size is selected so that representative elementary volumes (REV) are observed for all quantities of interest [39].

One thousand six hundred and eighty eight generation pairs consisting of forty one values of the length scale $\lambda$, ranging from $2.50 \cdot 10^{-5} \mathrm{~m}^{2}$ to $2.025 \cdot 10^{-3} \mathrm{~m}^{2}$ in increments of $5.00 \cdot 10^{-5} \mathrm{~m}^{2}$, and forty one values of the level threshold $\gamma$, ranging from 0.30 to 0.70 in increments of $1.0 \cdot 10^{-2}$, are considered. One-hundred and twenty eight realizations are produced using each generation pair. In sum, a total of 215618 realizations are generated and their physical characteristics analyzed. The lower end of the $\lambda$ values is selected because its square root, the standard deviation of the Gaussian kernel, is one order of magnitude larger than the computational grid spacing, and is well resolved. Above these values of $\lambda$, correlations in the topography are larger than the selected domain size. Such realizations do not produce an REV of the Minkowski functionals 
that are desired. The range of $\gamma$ is selected to create pore structures whose expected porosities range between zero and one.

For each realization, the first three Minkowski functionals for a porous sample (porosity, specific surface area, and mean curvature), and topological properties (number of pores and local coordination number) are computed. The fourth Minkowski functional, referred to as the Euler characteristic, is a measure of the connectivity of the pore space. The topological properties that are reported serve as an alternative measure for this functional.

The average value of these observables is computed for each generation pair. For a given generation pair, the average value of these observables approximates the expected value of that quantity. This computational investigation provides a table which can be used to select generation paris to create porous microstructures with desired values of the Minkowski functionals and topological properties. We illustrate this procedure in Section 5 where a realization is generated to have similar values of the Minkowski functionals as a imaged sample of Berea sandstone.

\subsection{Minkowski Functionals}

To provide a detailed description of how the geometric characteristics of the porous microstructures depend on a generation pair, we use the three Minkowski functionals for a porous medium to describe a realization $[16,17,18,38]$. For completeness, we recount the definitions of the four Minkowski functionals of a three dimensional porous sample and a description of their physical significance. For a complete treatment of the Minkowski functionals and their application to porous samples see [27]. The first functional of a porous medium $\mathbb{S}$ is porosity,

$$
\phi=V_{3}(\mathbb{P}) / V_{3}(\mathbb{S})=\mathbb{V}_{P} / \mathbb{V}_{\mathbb{S}} .
$$

Here $V_{d}(\mathbb{K})$ denotes the $d$-dimensional Lebesgue volume of an integrable set $\mathbb{K}, \mathbb{P}$ is the void space of the medium, $\mathbb{V}_{P}$ is the volume of the void space, and $\mathbb{V}_{\mathbb{S}}$ is the total volume of the realization $\mathbb{S}$. Physically, porosity is the ratio of the void volume over bulk volume and measures the relative capacity of a porous medium to hold water. The second functional, internal specific surface area,

$$
S S A=2 V_{2}(\mathbb{P}) / V_{3}(\mathbb{S})=2 \partial \mathbb{P} / \mathbb{V}_{\mathbb{S}} .
$$

is the total interstitial area between the pore space and the solid matrix. The boundary of the pore space $\partial \mathbb{P}$ is computed by counting the number of computational cell walls that separate solid and void nodes. Mean curvature,

$$
\mathcal{S}=V_{1}(\mathbb{P}) / V_{3}(\mathbb{S})=V_{1}(\mathbb{P}) / \mathbb{V}_{\mathbb{S}}
$$

is a measure of the average pore size of the sample over the total volume. Formally, $V_{1}(\mathbb{P})$ is mean value of the distance between a pair of parallel support planes. It is computed by counting the number of consecutive computational grid nodes in the void separating two computational grid nodes in the solid phase (or separating one solid computational grid node and a computational grid node on the block faces) along each of three Cartesian axis, as in [37]. This is equivalent to the definition of chord length provided by Torquato and $\mathrm{Lu}[40]$. There is also a fourth Minkowski functional $V_{0}(\mathbb{P}) / V_{3}(\mathbb{S})$, the Euler characteristic, that measures the connectivity of the pore space. Instead of this functional, we use other topological metrics that are easier to measure, which are described in Sec. 3.2.

\subsubsection{Example: Control of Minkowski Functionals}

To illustrate how the pore structure and Minkowski functionals depend on the selected generation pair, nine different generation pairs were used to create realizations from the same underlying random field, and horizontal cross sections of the three dimensional porous microstructures are shown in Fig. 2 where the solid phase is shown in black. The values of the first three Minkowski functionals are provided in Table 1. As $\lambda$ increases (top to bottom), the correlation length in the topography increases and larger values of porosity and mean curvature are observed. As $\gamma$ increases (left to right), more of the topography is mapped into the void space, thereby increasing the porosity and the mean curvature of the realizations. The specific surface areas decrease with longer correlation lengths, and are roughly symmetric about $\gamma=0.50$. 


\begin{tabular}{|c|c|c|c|c|c|c|c|c|c|}
\hline \multirow[b]{2}{*}{$\lambda$} & \multicolumn{3}{|c|}{$\gamma: 0.45$} & \multicolumn{3}{|c|}{$\gamma: 0.50$} & \multicolumn{3}{|c|}{$\gamma: 0.55$} \\
\hline & $\phi$ & $S S A$ & $\mathcal{S}$ & $\phi$ & $S S A$ & $\mathcal{S}$ & $\phi$ & $S S A$ & $\mathcal{S}$ \\
\hline $1.00 \cdot 10^{-4}$ & 0.28 & 0.86 & 0.17 & 0.45 & 1.02 & 0.24 & 0.64 & 0.97 & 0.36 \\
\hline $6.25 \cdot 10^{-4}$ & 0.32 & 0.38 & 0.37 & 0.48 & 0.42 & 0.54 & 0.64 & 0.39 & 0.81 \\
\hline $1.60 \cdot 10^{-3}$ & 0.30 & 0.23 & 0.49 & 0.44 & 0.26 & 0.70 & 0.59 & 0.26 & 1.01 \\
\hline
\end{tabular}

Table 1: Values of the Minkowski functionals for the realizations whose horizontal cross sections are shown in Fig. 2. Porosity $\phi$, specific surface area $S S A\left[\delta x^{-1}\right]$, and mean curvature, $\mathcal{S}\left[10^{-5} \delta x^{-2}\right]$ for the realizations whose cross sections are shown in Fig. 2. All realizations are generated using the same underlaying random field $u$.

(a) $\lambda=1.00 \cdot 10^{-4} \mathrm{~m}^{2}: \gamma=0.45$

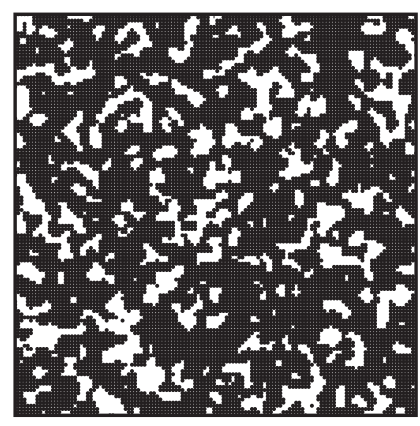

(d) $\lambda=6.25 \cdot 10^{-4} \mathrm{~m}^{2}: \gamma=0.45$



(g) $\lambda=1.60 \cdot 10^{-3} \mathrm{~m}^{2}: \gamma=0.45$

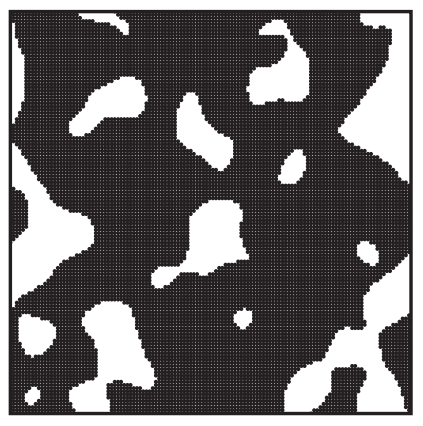

(b) $\lambda=1.00 \cdot 10^{-4} \mathrm{~m}^{2}: \gamma=0.50$

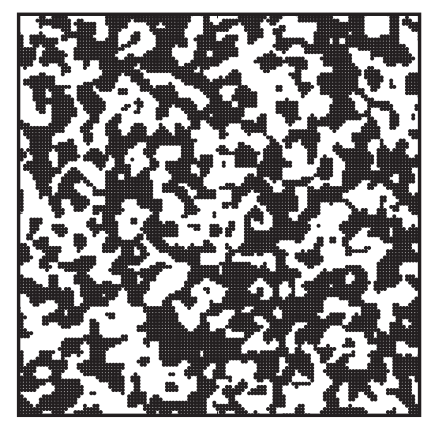

(e) $\lambda=6.25 \cdot 10^{-4} \mathrm{~m}^{2}: \gamma=0.50$

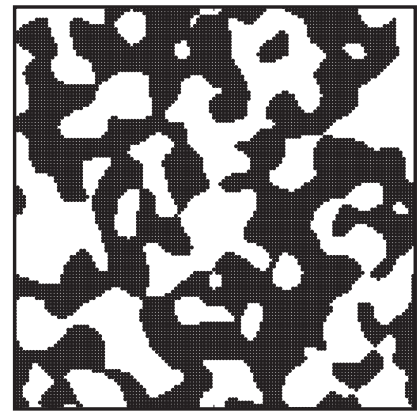

(h) $\lambda=1.60 \cdot 10^{-3} \mathrm{~m}^{2}: \gamma=0.50$



(c) $\lambda=1.00 \cdot 10^{-4} \mathrm{~m}^{2}: \gamma=0.55$

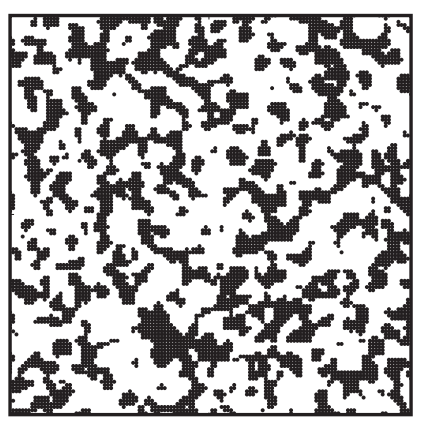

(f) $\lambda=6.25 \cdot 10^{-4} \mathrm{~m}^{2}: \gamma=0.55$



(i) $\lambda=1.60 \cdot 10^{-3} \mathrm{~m}^{2}: \gamma=0.55$

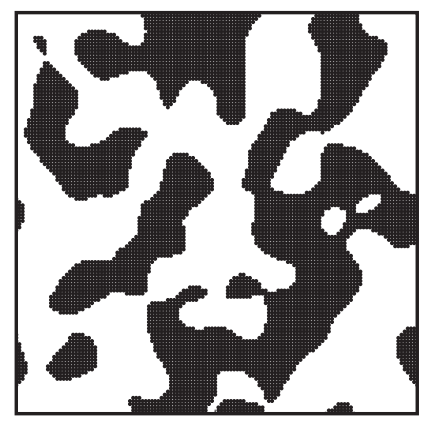

Fig. 2: Horizontal cross sections of virtual pore spaces generated from the same underlaying field with different generation pairs. The solid matrix is shown in black. The values of the first three Minkowski functionals are provided in Table 1.

\subsubsection{Average Minskowski Functional Values}

Contour plots of the computationally determined average values for porosity, specific surface area, and mean curvature are shown in Fig. 3, and values are given in Table 2 for select generation pairs. Using the information provided in Table 2, appropriate generation pairs can be selected to produce realizations with desired porosity, specific surface area, and mean curvature. 
(a) Porosity

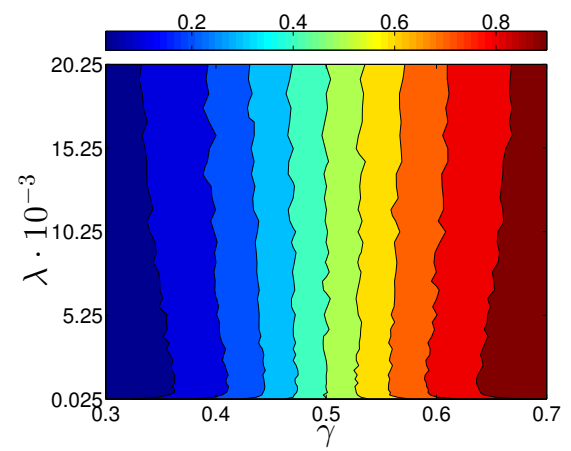

(b) Specific Surface Area

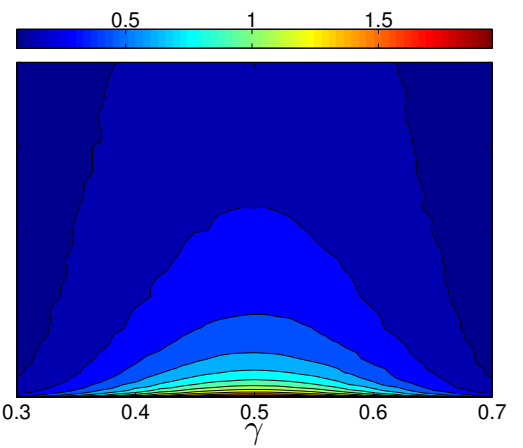

(c) Mean Curvature



Fig. 3: Contour plots of the average first three Minkowski Functionals - (a) Porosity, (b)Specific Surface Area [ $\left.\delta x^{-1}\right]$, (c) Mean Curvature $\left[10^{-5} \cdot \delta x^{-2}\right]$ - are plotted as functions of the generation parameters $\lambda$ and $\gamma$. All of the Minkowski functionals depend nonlinearly on the choice of $\lambda$ and $\gamma$. Values of the Minkowski functionals at select generation pairs are provided in Table 2.

The values of average porosity $\phi$ reported in Table 2 and shown in Fig. 3a indicate that at a fixed length scale $\lambda$, the average porosity rises with the level threshold $\gamma$. This relationship agrees with both intuition, as $\gamma$ increases, the probability that a point in the topography is less than $\gamma$ decreases, and the analytic expression for expected porosity (11). The relationship between $\gamma$ and the average porosity is linear over the considered range of $\gamma$; the correlation coefficient between the $\gamma$ values and average porosity is 0.99 . At a fixed value of $\gamma$, only slight variations among the average porosities is observed as $\lambda$ varies. This invariance is the result of mapping the topography $T$ such that $\min (T)=0$ and $\max (T)=1$. As previously mentioned, this mapping mitigates the degeneration of the topography to the mean value of the random field while preserving the correlation structure induced by the kernel. However, the analytic expression for porosity (11) does not take this remapping into account. Therefore an empirical model to predict the porosity of these mapped pore spaces is determined, namely

$$
\phi(\gamma, \lambda)=1 / 2+1 / 2 \operatorname{Erf}[a \sqrt{\lambda}+b(\gamma-1 / 2)]
$$

where the parameters $a$ and $b$ are included to account for the remapping of the topography. The coefficients (with $95 \%$ confidence bounds) are $a=0.013(-0.049,0.074)$ and $b=5.98(5.96,6.01)$. The value of $a \sim 0$ demonstrates how the remapping removes the influence of the characteristic length scale. The value of $b \sim 6$ combines the scaling coefficients in (11). The coefficient of determination $\left(R^{2}\right)$ is 0.9979 indicating that the empirical model fits the data well.

The average specific surface area, $S S A$, reported in Table 2 and shown in Fig. 3b indicates that $S S A$ depends nonlinearly on both $\gamma$ and $\lambda$. At a fixed length of $\lambda$, the values of $S S A$ are nearly symmetric about $\gamma=0.50$, rising with $\gamma$ from 0.30 to 0.50 , and decreasing when $\gamma$ increases from 0.50 to 0.70 . Once the volume of the solid phase and void space are equal, $\phi=0.50$, the volume of the solid phase decreases and so does the amount of exposed surface area. On the other hand, at a fixed level of $\gamma$ the surface area decreases exponentially as $\lambda$ increases. The values reported for the shortest length scale, $\lambda=2.50 \cdot 10^{-5}$, are much larger than those recorded for any other value of $\lambda$. This shorter length scale creates a more rugged topography, which results in numerous narrow pores. Physically, the topographies generated with longer correlation lengths create realizations with wider pores which have less specific surface area than realizations generated using smaller values of $\lambda$. The average specific surface area is well modeled by the function

$$
S S A(\gamma, \lambda)=\delta x^{-1} \exp \left[a(\gamma-1 / 2)^{2}+b \lambda^{-1 / 8}+c\right] .
$$

The coefficients (with 95\% confidence bounds) are $a=-38.3(-38.59,-38) b=1.32(1.315,1.324)$ and $c=$ $9.653(9.637,9.669)$ and the coefficient of determination $\left(R^{2}\right)$ is 0.9948.

The average values of mean curvature, $\mathcal{S}$, reported in Table 2 and shown in Fig. $3 c$ indicates that the typical pore size of a realization depends on both $\lambda$ and $\gamma$. Larger values of $\lambda$ create longer correlations in 
the topography which produce wider pores once $\gamma$ is applied. Nonetheless, as $\gamma$ rises, the width of pores increases as well. At a fixed level of $\lambda$, the pore sizes are well modeled by a power law where the exponent of $\gamma$ depends upon the particular kernel width. Similar behavior is observed at a fixed level of $\gamma$, where the pore sizes also follow a power law where the exponent of $\lambda$ depends on the particular level of $\gamma$. An empirical model that combines the generation pair into a sum of power laws,

$$
\mathcal{S}(\gamma, \lambda)=\delta x^{-2}\left(a \gamma^{b}+c \lambda^{d}\right)
$$

of $\mathcal{S}$, fits the data with coefficients (with $95 \%$ confidence bounds) of $a=932.2(846.2,1018) \mathrm{b}=7.276$ $(7.039,7.514), \mathrm{c}=4427(1307,7547)$, and $d=0.9196(0.8097,1.029)$ and the coefficient of determination $\left(R^{2}\right)$ is 0.9218 . At low values of $\lambda, \lambda \leq 2.50 \cdot 10^{-5}$, the data does not follow a power law and the model performs poorly, cf. Table 2 . The rugged topography created by these narrow kernels produces narrow pores once the threshold is applied. Thus, the change in pore radius between two different narrow kernels is more than that for wider kernels. Restricting the fitting to values of $\lambda \geq 2.25 \cdot 10^{-4}$, the $95 \%$ confidence bounds tighten, and the $R^{2}$ value increases to 0.9833. Furthermore, using these limited values of $\lambda$ the exponent of $\lambda, d=1.019(0.8946,1.143)$, suggesting a linear dependence of mean curvature on $\lambda$, which is also observed in both Fig. 3c and Table 2. These power law exponents suggest that $\gamma$ controls the mean curvature more than $\lambda$, even though $\lambda$ determines the correlation structure of $T$.

\begin{tabular}{|c|c|c|c|c|c|c|c|c|c|}
\hline \multirow[b]{2}{*}{$\lambda$} & \multicolumn{3}{|c|}{$\gamma: 0.30$} & \multicolumn{3}{|c|}{$\gamma: 0.35$} & \multicolumn{3}{|c|}{$\gamma: 0.40$} \\
\hline & $\phi$ & $S S A$ & $\mathcal{S}$ & $\phi$ & $S S A$ & $\mathcal{S}$ & $\phi$ & $S S A$ & $\mathcal{S}$ \\
\hline $2.50 \cdot 10^{-5}$ & 0.07 & 0.56 & 0.06 & 0.14 & 1.03 & 0.07 & 0.24 & 1.51 & 0.09 \\
\hline $2.25 \cdot 10^{-4}$ & 0.04 & 0.13 & 0.09 & 0.09 & 0.26 & 0.12 & 0.18 & 0.45 & 0.18 \\
\hline $6.25 \cdot 10^{-4}$ & 0.04 & 0.09 & 0.11 & 0.10 & 0.18 & 0.17 & 0.20 & 0.29 & 0.26 \\
\hline $1.23 \cdot 10^{-3}$ & 0.05 & 0.08 & 0.13 & 0.12 & 0.14 & 0.22 & 0.21 & 0.21 & 0.33 \\
\hline \multirow[t]{2}{*}{$2.03 \cdot 10^{-3}$} & 0.06 & 0.07 & 0.16 & 0.13 & 0.12 & 0.26 & 0.22 & 0.17 & 0.40 \\
\hline & \multicolumn{3}{|c|}{$\gamma: 0.45$} & \multicolumn{3}{|c|}{$\gamma: 0.50$} & \multicolumn{3}{|c|}{$\gamma: 0.55$} \\
\hline$\lambda$ & $\phi$ & $S S A$ & $\mathcal{S}$ & $\phi$ & $S S A$ & $\mathcal{S}$ & $\phi$ & $S S A$ & $\mathcal{S}$ \\
\hline $2.50 \cdot 10^{-5}$ & 0.36 & 1.88 & 0.11 & 0.50 & 2.03 & 0.14 & 0.63 & 1.89 & 0.19 \\
\hline $2.25 \cdot 10^{-4}$ & 0.32 & 0.61 & 0.25 & 0.50 & 0.68 & 0.38 & 0.68 & 0.61 & 0.62 \\
\hline $6.25 \cdot 10^{-4}$ & 0.33 & 0.37 & 0.38 & 0.48 & 0.41 & 0.56 & 0.66 & 0.38 & 0.87 \\
\hline $1.23 \cdot 10^{-3}$ & 0.34 & 0.27 & 0.50 & 0.51 & 0.29 & 0.75 & 0.67 & 0.27 & 1.18 \\
\hline \multirow[t]{2}{*}{$2.03 \cdot 10^{-3}$} & 0.35 & 0.21 & 0.60 & 0.50 & 0.23 & 0.85 & 0.65 & 0.21 & 1.36 \\
\hline & \multicolumn{3}{|c|}{$\gamma: 0.60$} & \multicolumn{3}{|c|}{$\gamma: 0.65$} & \multicolumn{3}{|c|}{$\gamma: 0.70$} \\
\hline$\lambda$ & $\phi$ & $S S A$ & $\mathcal{S}$ & $\phi$ & $S S A$ & $\mathcal{S}$ & $\phi$ & $S S A$ & $\mathcal{S}$ \\
\hline $2.50 \cdot 10^{-5}$ & 0.76 & 1.52 & 0.28 & 0.86 & 1.02 & 0.47 & 0.93 & 0.58 & 0.97 \\
\hline $2.25 \cdot 10^{-4}$ & 0.82 & 0.45 & 1.05 & 0.92 & 0.26 & 1.87 & 0.97 & 0.12 & 3.40 \\
\hline $6.25 \cdot 10^{-4}$ & 0.80 & 0.29 & 1.50 & 0.91 & 0.17 & 2.50 & 0.96 & 0.09 & 3.73 \\
\hline $1.23 \cdot 10^{-3}$ & 0.78 & 0.22 & 1.77 & 0.89 & 0.14 & 2.82 & 0.95 & 0.08 & 3.97 \\
\hline $2.03 \cdot 10^{-3}$ & 0.77 & 0.17 & 2.05 & 0.87 & 0.12 & 2.98 & 0.94 & 0.07 & 3.99 \\
\hline
\end{tabular}

Table 2: Computationally determined average values of the Minkowski functionals, porosity $\phi$, specific surface area $S S A\left[\delta x^{-1}\right]$, and mean curvature, $\mathcal{S}\left[10^{-5} \delta x^{-2}\right]$.

\subsection{Pore Topology}

We investigate how generation pairs determine the topology of these pore space realizations by extracting a network representation of the void space using a maximal ball algorithm to extract graphs of pore networks from binary images [41]. The algorithm divides the void space of the pore structure into two classes, pores, which are larger regions in the void space, and throats, which connect the pores together. The result of applying the algorithm is a connected graph with a set of vertices, pores in the network, and edges, throats which connect pores. The degree of each vertex in the graph corresponds to the coordination number of that pore. The average number of pores, $N_{p}$, average coordination number, $C N$, and the ratio of average coordination number over the average number of pores minus one, $r=C N /\left(N_{p}-1\right)$, for the realization sets is provided in Table 3 . 
Similar to the Minkowski functionals, these topological properties depend nonlinearly upon the generation pair. Small values of $\lambda$ create realizations with more pores than those generated by kernels with longer length scales. Recall that there is little variation in the expected porosity at a given level of $\gamma$ (Table 2). In other words, there is little variation in the void volume at a fixed level of gamma. This constant void volume along with the decrease in $N_{P}$, indicates that the pore volume increases with increasing values of $\lambda$. On the other hand, as $\gamma$ increases the number of pores steadily decreases. This behavior is not observed in the narrowest kernel, likely a result of the more variable topography that the narrow kernel induces. The narrow kernel results in more small pores as $\gamma$ increases up to 0.45 . After this value, the smaller pores connect to create larger, although fewer, pores.

\begin{tabular}{|c|c|c|c|c|c|c|c|c|c|}
\hline \multirow[b]{2}{*}{$\lambda$} & \multicolumn{3}{|c|}{$\gamma: 0.30$} & \multicolumn{3}{|c|}{$\gamma: 0.35$} & \multicolumn{3}{|c|}{$\gamma: 0.40$} \\
\hline & $N_{p}$ & $C N$ & $r$ & $N_{p}$ & $C N$ & $r$ & $N_{p}$ & $C N$ & $r$ \\
\hline $2.50 \cdot 10^{-5}$ & 123.88 & 0.10 & 0.00 & 1027.44 & 1.42 & 0.00 & 4540.33 & 5.88 & 0.00 \\
\hline $2.25 \cdot 10^{-4}$ & 817.98 & 0.52 & 0.00 & 1319.45 & 1.18 & 0.00 & 1507.73 & 2.58 & 0.00 \\
\hline $6.25 \cdot 10^{-4}$ & 325.61 & 0.70 & 0.00 & 425.38 & 1.34 & 0.00 & 442.01 & 2.43 & 0.01 \\
\hline $1.23 \cdot 10^{-3}$ & 143.97 & 0.75 & 0.01 & 183.26 & 1.45 & 0.01 & 184.62 & 2.54 & 0.01 \\
\hline $2.03 \cdot 10^{-3}$ & 80.99 & 0.89 & 0.01 & 96.34 & 1.55 & 0.02 & 96.50 & 2.66 & 0.03 \\
\hline & \multicolumn{3}{|c|}{$\gamma: 0.45$} & \multicolumn{3}{|c|}{$\gamma: 0.50$} & \multicolumn{3}{|c|}{$\gamma: 0.55$} \\
\hline$\lambda$ & $N_{p}$ & $C N$ & $r$ & $N_{p}$ & $C N$ & $r$ & $N_{p}$ & $C N$ & $r$ \\
\hline $2.50 \cdot 10^{-5}$ & 7600.49 & 8.93 & 0.00 & 5762.59 & 11.68 & 0.00 & 4434.41 & 13.22 & 0.00 \\
\hline $2.25 \cdot 10^{-4}$ & 1273.85 & 4.89 & 0.00 & 857.52 & 7.93 & 0.01 & 480.48 & 10.91 & 0.02 \\
\hline $6.25 \cdot 10^{-4}$ & 378.50 & 4.26 & 0.01 & 255.39 & 6.82 & 0.03 & 147.81 & 9.12 & 0.06 \\
\hline $1.23 \cdot 10^{-3}$ & 159.60 & 4.06 & 0.03 & 105.60 & 6.30 & 0.06 & 66.05 & 7.72 & 0.12 \\
\hline $2.03 \cdot 10^{-3}$ & 80.90 & 4.09 & 0.05 & 59.89 & 5.58 & 0.09 & 37.85 & 6.66 & 0.18 \\
\hline & \multicolumn{3}{|c|}{$\gamma: 0.60$} & \multicolumn{3}{|c|}{$\gamma: 0.65$} & \multicolumn{3}{|c|}{$\gamma: 0.70$} \\
\hline$\lambda$ & $N_{p}$ & $C N$ & $r$ & $N_{p}$ & $C N$ & $r$ & $N_{p}$ & $C N$ & $r$ \\
\hline $2.50 \cdot 10^{-5}$ & 2167.19 & 14.62 & 0.01 & 1294.90 & 16.21 & 0.01 & 682.08 & 16.87 & 0.02 \\
\hline $2.25 \cdot 10^{-4}$ & 240.73 & 12.79 & 0.05 & 112.85 & 13.01 & 0.12 & 53.34 & 12.12 & 0.23 \\
\hline $6.25 \cdot 10^{-4}$ & 75.31 & 10.17 & 0.14 & 40.61 & 9.99 & 0.25 & 17.41 & 8.19 & 0.50 \\
\hline $1.23 \cdot 10^{-3}$ & 38.16 & 8.23 & 0.22 & 19.41 & 7.57 & 0.41 & 9.36 & 5.82 & 0.70 \\
\hline $2.03 \cdot 10^{-3}$ & 23.09 & 6.81 & 0.31 & 13.21 & 6.03 & 0.49 & 6.38 & 4.65 & 0.86 \\
\hline
\end{tabular}

Table 3: Average Pore Space Topology Properties : Number of Pores $N_{p}$, Coordination Number $C N$ of each pore, and the percentage of connectivity $r$. The global percentage of connectivity rises with kernel length $\lambda$ and level threshold $\gamma$.

Larger values of the average coordination number $C N$ imply that the pore space is locally well-connected, i.e., a typical pore is directly linked to a larger number of other pores. At a fixed level of $\gamma$, the values of the $C N$ decrease slightly as $\lambda$ increases. At all values of $\lambda$, the coordination number increases slightly as the threshold level rises to $\gamma=0.60$ and decreases afterword. At low values of $\gamma$, a typical pore has between one and two neighbors, and at higher values of $\gamma$ it can have up to sixteen neighbors. One can visualize these results by thinking about the correlated topography as a landscape and $\gamma$ as water level. At low water levels the pores correspond to sparsely connected lakes in valleys of the topography. As the water level rises the lakes increase their connectivity via saddles between high regions of the topography until the topography is filled by a few large lakes.

The quantity $r=C N /\left(N_{p}-1\right)$ measures the global connectivity of the pore network. If the value of $r$ is near one, then the network is globally well-connected; a typical pore is directly linked to a large fraction of the network. If the value of $r$ is near zero, then the network is globally poorly connected; a typical pore is directly linked to a small fraction of the network. The reported values in Table 3 indicate that the global connectivity varies considerably with generation pair, even though the coordination numbers $C N$ only vary by a factor of 2-3 across kernel widths. At the lowest level of $\gamma$, most pores are directly connected to less than one percent of the network. However, as $\gamma$ and $\lambda$ increase,the average pore is connected to a larger percentage of the network. For a fixed value of $\gamma, r$ increases with $\lambda$ indicating that pore spaces generated with wider kernels are more globally connected than those generated with narrow ones.

The relationship between connectivity, kernel width, and the level threshold agrees with physical intuition. Longer correlations in the topography induced by wider kernel widths result in larger pores, and as the 
level threshold rises more of the topography is mapped into the void space. These few wide pores connect to one another as the level threshold rises. At the same time, these results do not indicate that pore spaces generated with narrow kernels and low threshold levels are poorly connected. On the contrary, smaller more numerous pores are more locally connected (Table 3). Using a breadth-first connected components algorithm it was determined that the void space is primarily a single connected component when the value of $\gamma$ is greater than 0.35 , regardless of kernel width.

The primary application of these pore space realizations is to better understand how pore structures influence flow and transport properties. Therefore, it is imperative that the void space connects from the top of the realization to the bottom, i.e., percolates. Intuitively, the probability that percolation occurs increases with rising level threshold $\gamma$. Using a breadth-first connected components algorithm it was determined that percolation does not occur for $\gamma$ values less than 0.28 , regardless of kernel width. Once $\gamma$ is greater than 0.46 , percolation almost surely occurs. The exact value of $\gamma$ such that percolation occurs depends upon the value of $\lambda$, the choice of kernel, and the dimensionality of the problem.

\section{Extensions}

In this section we describe two extensions of the method to produce diverse porous structures. The first introduces anisotropy into the pore space to create preferential flow directions, and the second, generates samples with multiple pore structures.

\subsection{Anisotropy}

Anisotropy can be introduced into a realization by using an asymmetric kernel in the convolution to produce longer correlations in the topography $T$ in desired directions. Once the level threshold $\gamma$ is applied, the longer correlations result in wider pores and create preferential flow directions through the resulting pore-space. To illustrate this extension, three realizations are produced by convolving the same random field $u$ with one symmetric Gaussian kernel and two different asymmetric Gaussian kernels. We use the techniques of Smolarkiewicz and Winter [1] to numerically integrate the Navier-Stokes equations using the EULAG system [42] and determine steady state fluid velocity fields within the three realizations. The flow solutions demonstrate how the anisotropy of the pore spaces influences the velocity field in the pore space.

In all three realizations, the length scales, $\lambda_{x}, \lambda_{y}$, and $\lambda_{z}$, are aligned with the Cartesian axis. In the first realization, the kernel is symmetric and the three length scales are equal, $\lambda_{x}=\lambda_{y}=\lambda_{z}=1.00 \cdot 10^{-4} \mathrm{~m}^{2}$. In the second, $\lambda_{x}$ and $\lambda_{y}$, are equal to four times the value of $\lambda_{z}, \lambda_{x}=\lambda_{y}=4 \cdot \lambda_{z}$, which remains at $1.00 \cdot 10^{-4} \mathrm{~m}^{2}$. In the third realization, the length scales in the horizontal directions, $\lambda_{x}$ and $\lambda_{y}$, are equal to $1.00 \cdot 10^{-4} \mathrm{~m}^{2}$ and the value of $\lambda_{z}$ is four times that value, $\lambda_{x}=\lambda_{y}=1 / 4 \cdot \lambda_{z}$. The same value of $\gamma$ is used to produce the final pore space realization from each topography, $\gamma=0.45$. Each pore-space has dimensions $L_{x}=L_{y}=L_{z}=1.27 \cdot 10^{-2} \mathrm{~m}$, with volume $V=2.05 \cdot 10^{-6} \mathrm{~m}^{3}$, and the domain is discretized using a uniform Cartesian grid with a grid spacing of $\delta x=10^{-4} \mathrm{~m}$.

Values of the Minkowski functionals, Sec. 3.1, for the three samples are reported in Table 4. The porosities $\phi$ of the three samples are approximately the same, $\phi \sim 0.30$. The two anisotropic samples have lower values of specific surface areas $S S A$ than the isotropic sample, which is consistent with the computational investigation where longer correlations appear to create samples with lower $S S A$, cf. Sec 3.1.2. The values of mean curvature are computed in each of the three Cartesian coordinates $\left(\mathcal{S}_{x}, \mathcal{S}_{y}\right.$, and $\left.\mathcal{S}_{z}\right)$ and correspond to the average pore radius in each direction. The pore sizes are approximately equal in the realization generated using the symmetric kernel, kernel (a). The influence of the asymmetric kernels is most notable in the reported values of the mean curvature in the anisotropic samples. The topography of the first anisotropic realization, kernel (b), has longer correlations in the horizontal plane that result in wider pores in $x$ and $y$. Similarly, the second anisotropic realization, kernel (c), was created by thresholding the topography with longer correlations in the vertical plane, which results in wider pores in the $z$ direction.

To demonstrate how the anisotropy influences the flow of fluids through these realizations, the methods of Smolarkiewicz and Winter [1] are used to simulate gravity driven laminar flow of an isothermal, single phase fluid within the three pore spaces. The multi-scale computational fluid model EULAG [42], is used 


\begin{tabular}{c|c|c|c|c|c|c|c} 
Kernel & $\phi$ & $S S A$ & $\mathcal{S}_{x}$ & $\mathcal{S}_{y}$ & $\mathcal{S}_{z}$ & $\tau$ & $\mathcal{T}$ \\
\hline (a) & 0.30 & 1.78 & $1.75 \cdot 10^{-6}$ & $1.74 \cdot 10^{-6}$ & $1.84 \cdot 10^{-6}$ & 1.38 & 0.65 \\
(b) & 0.33 & 1.24 & $3.21 \cdot 10^{-6}$ & $3.19 \cdot 10^{-6}$ & $1.99 \cdot 10^{-6}$ & 1.78 & 0.67 \\
(c) & 0.28 & 1.43 & $1.67 \cdot 10^{-6}$ & $1.68 \cdot 10^{-6}$ & $3.37 \cdot 10^{-6}$ & 1.22 & 0.38
\end{tabular}

Table 4: Pore space and flow statistics for anisotropic pore spaces and control pore space. (a) Symmetric Kernel (b) Correlation in the direction perpendicular to flow is four times that in the direction in the primary direction of flow (c) Correlation in the primary direction of flow is four times that perpendicular to flow. Porosity $\phi$, Specific Surface Area, $S S A\left[\delta x^{-1}\right.$ ], Mean curvature in $x, \mathcal{S}_{x}\left[\delta x^{-2}\right]$, Mean curvature in $y, \mathcal{S}_{y}\left[\delta x^{-2}\right]$, Mean curvature in $z \mathcal{S}_{z}\left[\delta x^{-2}\right]$, Mean tortuosity $\tau$, Mean passage time $\mathcal{T}[\mathrm{sec}]$.

(a) $\lambda_{x}=\lambda_{y}=\lambda_{z}$

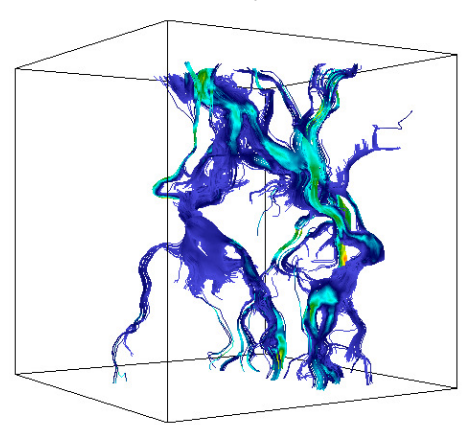

(b) $\lambda_{x}=\lambda_{y}=4 \lambda_{z}$



(c) $\lambda_{x}=\lambda_{y} ; \lambda_{z}=4 \lambda_{x}$

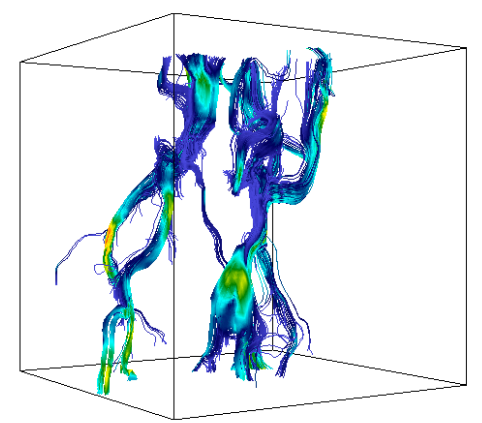

Fig. 4: Particle trajectories in pore spaces generated from the same underlaying random field with different kernel symmetries. The threshold is fixed at $\gamma=0.45$. Subplot (a) Symmetric Kernel (b) Longer correlation in the direction perpendicular to flow (horizontal) (c) Longer correlation in the primary direction of flow (vertical)

to numerically integrate the Navier-Stokes equations from rest until steady state conditions are reached. No-slip boundary conditions are enforced along pore walls using an immersed-boundary method (IB) [43, $44,45,46,47]$. The IB originated in the area of computational biomechanics [48, 49], and employs fictitious body forces in the equations of motion to mimic the presence of solid structures and internal boundaries; see [50] for a review. The particular technique adapted is a variant of feedback forcing [51], with implicit time discretization admitting rapid attenuation of the flow to stagnation (within solid structures) in $\mathcal{O}(\Delta t)$ time comparable to the time step $\Delta t$ of the fluid model. The efficacy of the IB in EULAG has been verified by comparisons with solutions using boundary fitted coordinates and wind tunnel data [46]. Each medium is periodic in the vertical direction with no flow boundary conditions applied along lateral boundaries. The flow simulations are run for $5 \times 10^{-2}$ seconds with steady state conditions reached in $2-3 \times 10^{-2}$ seconds.

Figure 4 shows a selection of particle trajectories, visualized using the stream-tracer module in Paraview [52], in the steady state velocity fields within each realization. The primary direction of flow is from top to bottom and the trajectories are colored according to normalized velocity magnitude, warmer colors indicating larger values. Trajectories in the isotropic realization, Fig. 4a, provide a control setting for the other two subfigures. Trajectories in the first anisotropic realization, Fig. 4b, show that the decreased resistance in the horizontal directions, perpendicular to the primary direction of flow, results in more tortuous fluid particle trajectories. The trajectories in the second anisotropic realization, Fig. 4c, show that the decreased resistance in the vertical direction, parallel to the primary direction of flow, results in straighter trajectories.

Mean tortuosity $\tau$ and mean passage time $\mathcal{T}$ of fluid particle trajectories are computed using the techniques of Hyman et al. [3] (Table 4). The adopted definition of tortuosity is the ratio of a particle's trajectory length over the distance between its endpoints. It measures the deviation of a trajectory from a straight-line path, high values indicating more tortuous paths. The values of tortuosity reported in Table 4, demonstrate how the trajectories in the anisotropic realizations differ from those passing through the isotropic realization. The trajectories in the realization with longer correlation in the horizontal plane, kernel (b), are more tortuous than the trajectories in the isotropic realization, as observed in Fig. 4b. On the other hand, the trajectories in the realization with longer pores in the vertical direction, kernel (c), are less tortuous than 
(a) $\chi_{1}(\mathbf{x})$

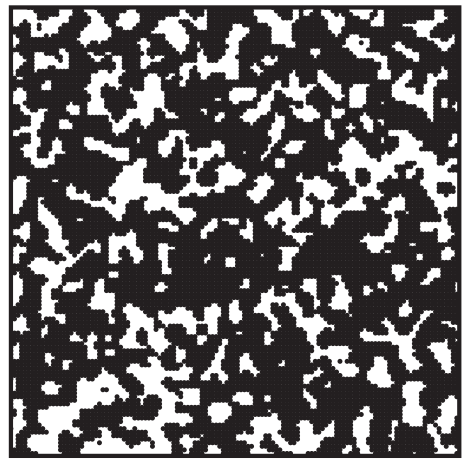

(b) $\chi_{2}(\mathbf{x})$



(c) $\chi_{3}(\mathbf{x})$

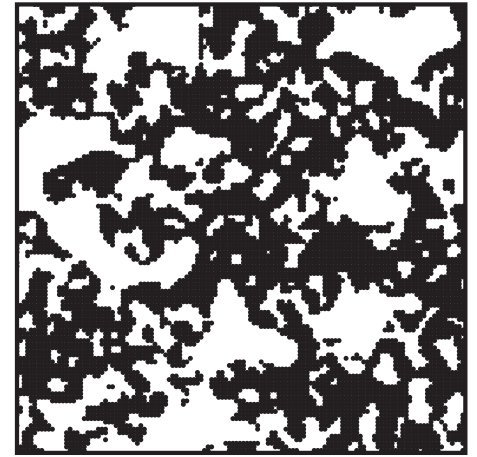

Fig. 5: Horizontal cross sections of three pore spaces. The pore space on the right is the point wise product of the left and middle pore spaces and contains the pore space of both its parent realizations.

the trajectories in the isotropic realization due to the decreased resistant to flow in the vertical direction, as observed in Fig. 4c. The mean travel time for the set of fluid particles is the average time it takes for all percolating particles to pass through the entire domain. Low passage times indicate fast trajectories, and large passage times indicate slow ones. Compared to the isotropic realization, the trajectories passing through the realization with wider pores in the horizontal plane are slightly slower. On the other hand, the trajectories passing through the realization with longer correlations in the vertical direction are almost twice as fast as those in the isotropic sample.

\subsection{Multiple Pore Structures}

Most real rocks contain pores that exhibit multiple length scales, e.g., numerous small pores embedded in a solid phase that also contains a few large pores. Realizations containing multiple pore structures can be produced using this method by combining two realizations created with different generation pairs.

One way to create a realization with multiple pore structures is by embedding smaller pores into the solid phase of a realization with larger pores. Figure 5 provides an example of this procedure. First, two pore spaces, $\chi_{1}(\mathbf{x})$ and $\chi_{2}(\mathbf{x})$, are independently created using different generation pairs. Figure 5a is a horizontal cross-section of a realization generated to have narrow pores, $\chi_{1}(\mathbf{x})$ where $\gamma=0.45$ and $\lambda=1.00 \cdot 10^{-4} \mathrm{~m}^{2}$, and Fig. 5b is the horizontal cross section of an independent realization with wider pores, $\chi_{2}(\mathbf{x})$ where $\gamma=0.40$ and $\lambda=4.00 \cdot 10^{-4} \mathrm{~m}^{2}$. These realizations are multiplied together point-wise to produce a third realization $\chi_{3}^{*}(\mathbf{x})=\chi_{1}^{*}(\mathbf{x}) \odot \chi_{2}^{*}(\mathbf{x})$ which is shown in Fig. $5 \mathrm{c}$. Here $\odot$ denotes the point wise product and * transforms the indicator function (2) so that $\chi(\mathbf{x})=0$ if $\mathbf{x}$ is in the void space and $\chi(\mathbf{x})=1$ otherwise. Using this transformation, the point wise multiplication of the initial two realizations preserves the voids spaces of both samples, and the resulting pore structure contains the pores of both its parent realizations. Figure $5 \mathrm{c}$ illustrates how the resulting pore space contains both the smaller pores of the first pore space and the larger pores of the second. Any number of realizations can be combined in this way to produce a sample with multiple pore structures.

Realizations of non-stationary pore spaces consisting of two (or more) stationary spaces with a smooth transition between them can be derived by mixing pore spaces created independently using the basic method. Consider the combination of $T_{1}$ and $T_{2}$, two topographies, with different correlation structures. A nonstationary topography with a transition along a boundary, $\partial G_{1,2}$, can be created by combining $T_{1}$ and $T_{2}$ by means of a mixing function, $f(x)$. In one dimension, for instance, this could correspond to $T_{3}(x)=$ $T_{1}(x) f(x-\xi)+T_{2}(x) f(\xi-x)$, where $\xi$ is a point on $\partial G_{1,2}$, e.g. $f(x)=\pi^{-1} \arctan (\alpha x)+1 / 2$ or $f(x)=$ $\exp (\alpha x) /(1+\exp (\alpha x))$. After the new composite topography has been generated the level threshold can be applied to produce the final pore space. Figure 6 shows an example of one such composite medium. The topography used to generate the pore space shown in Fig. 6a was created using a Gaussian kernel with $\lambda=1.00 \cdot 10^{-4} \mathrm{~m}^{2}$ and the topography used to generate the pore space shown in Fig. $6 \mathrm{~b}$ was created using a 
(a) $\chi_{1}(\mathbf{x}) \lambda: 1.00 \cdot 10^{-4} \mathrm{~m}^{2}$



(b) $\chi_{2}(\mathbf{x}) \lambda: 4.00 \cdot 10^{-4} \mathrm{~m}^{2}$



(c) $\chi_{3}(\mathbf{x})$ Composite of $T_{1}$ and $T_{2}$



Fig. 6: Horizontal cross sections of three pore spaces. The topography used to generated the pore space on the right is a composite media generated using the topographies underlying the two pore spaces on the left.

Gaussian kernel with $\lambda=4.00 \cdot 10^{-4} \mathrm{~m}^{2}$. Figure $6 \mathrm{c}$ illustrates how the method produces a smooth transition between the first two pore spaces. A level threshold of $\gamma=0.50$ was used to produce all three pore spaces.

\section{Example : Berea Sandstone}

While the imaging of real rocks can generate high resolution images of pore spaces, the number of realizations that can be obtained is limited by the availability of samples, the costs of imaging, and errors that arise in image processing. These limitations make the investigation and quantification of a pore structure's influence on flow and transport using multiple independent samples infeasible. Therefore, researchers commonly assume the ergodic hypothesis and replace ensemble averages with spatial ones obtained using a few samples. However, virtual realizations do not suffer from these shortcomings because stochastic generation methods can produce numerous samples at low cost. To alleviate these issues the two methodologies are often combined. The parameters of the stochastic generation method are guided by an imaged pore space to produce virtual samples with similar characteristics. The metric used to define the represented characteristics is a major difference amongst stochastic generation methods.

The method presented in this paper provides a framework for the generation of pore space realizations based on the Minkowski functionals of a real pore space. To illustrate this ability, we create a realization based on the Minkowski functionals of an imaged sample of Berea sandstone ${ }^{1}$. An equivalence of the samples is validated by computing and comparing their permeabilities, a bulk hydrological property.

A cylindrical sample of Berea sandstone measuring $6.5 \mathrm{~mm}$ in diameter by $6.5 \mathrm{~mm}$ in length was imaged using five hundred and twelve images with a pixel size of $12.7 \mu \mathrm{m}$ using an X-ray Microscopic CT Scanner (Microscopic Scan Co., LTD, Japan). The CT scanner used in the study has a capacity for scanning a sample with maximum dimensions of $50 \mathrm{~mm}$ at a resolution of $1 \mu \mathrm{m}$. The sample was scanned under the conditions of $100 \mathrm{kV}$ and $0.020 \mathrm{~mA}$. The images of the sub-volume are thresholded to produce a binary representation of the pore space using the optimal thresholding technique [54]. A cubic sub-volume with dimensions $L_{x}=L_{y}=L_{z}=1.6 \mathrm{~mm}$ and volume $4.29 \cdot 10^{-9} \mathrm{~m}^{3}$, was extracted from the entire sample for this study. Figure 7a shows a horizontal cross section of the binary representation of the sub-volume. General information about Berea sandstone can be found in [55].

The Minkowski functionals and permeability of the sub-volume are reported in Table 5. Permeability measures a medium's macroscopic resistance to flow and is defined via Darcy's law in terms of the ratio of fluid flux to the gradient of pressure within the fluid. We determine the permeability of the sample computationally using the techniques of [5]. The values of the Minkowski functionals are compared to those in Table 2 to determine proper values of the generation pair, $\gamma$ and $\lambda$, to create a sample which reproduces

${ }^{1}$ Provided by Ming Zhang [53] 
the Minkowski functionals of the Berea. It appears that the Berea sample exhibits pore radii at two different length scales, one large and one small, and a slight anisotropy in the mean curvature. To account for these features, two media with different pore radii are created and combined using the technique described in Sec. 4 to create a realization with multiple pore structures, and a slight anisotropy is introduced into the realizations by using asymmetric Gaussian kernels to account for narrower pore radii in the vertical direction. One sample is generated using a threshold of $\gamma=0.33$ and length scale of $\lambda_{x}=\lambda_{y}=6.25 \cdot 10^{-5} \mathrm{~m}^{2}$, and a second is generated using a threshold of $\gamma=0.40$ and kernel length scale of $\lambda_{x}=\lambda_{y}=4.23 \cdot 10^{-5} \mathrm{~m}^{2}$; $\lambda_{z}=0.9 \cdot \lambda_{x}$ in both samples.

\begin{tabular}{c|cccccc} 
Samples & $\phi$ & $S S A$ & $\mathcal{S}_{x}$ & $\mathcal{S}_{y}$ & $\mathcal{S}_{z}$ & $\mathcal{K}$ \\
\hline Real & 0.21 & 0.46 & $1.22 \cdot 10^{-6}$ & $1.22 \cdot 10^{-6}$ & $1.17 \cdot 10^{-6}$ & 4.11 \\
Virtual & 0.22 & 0.48 & $1.24 \cdot 10^{-6}$ & $1.22 \cdot 10^{-6}$ & $1.19 \cdot 10^{-6}$ & 3.03
\end{tabular}

Table 5: Minkowski functionals and permeability of an imaged Berea sandstone and a sample generated to have the similar characteristics. Porosity $\phi$, Specific surface area $S S A\left[12.7 \mu \mathrm{m}^{-1}\right]$, Mean curvature in $x, \mathcal{S}_{x}\left[12.7 \mu \mathrm{m}^{-2}\right]$, Mean curvature in $y$, $\mathcal{S}_{y}\left[12.7 \mu \mathrm{m}^{-2}\right]$, Mean curvature in $z \mathcal{S}_{z}\left[12.7 \mu \mathrm{m}^{-2}\right]$, and permeability $\mathcal{K}$ [Darcies].

The Minkowski functionals and permeability of the realization created to mimic the sub-volume of the Berea are reported in Table 5 and Fig. 7b shows a horizontal cross section of the virtual sample beside the real sample. Their Minkowski functionals are close to one another, and the anisotropy of the mean curvature observed in the real sample is represented in the virtual realization. Moreover, the values of permeability, $\mathcal{K}$, are close to one another, a difference of $\sim 1$ Darcy, or $\sim 2 \cdot 10^{-12} \mathrm{~m}^{2}$. Hence, the virtual sample reproduces bulk hydrological properties of the real sample. The horizontal cross sections shown in Figure 7 demonstrate that the synthetic sample's multiple pore structures mimic those of the real sample.

(a) Real Berea Sandstone



(b) Synthetic Berea Sandstone

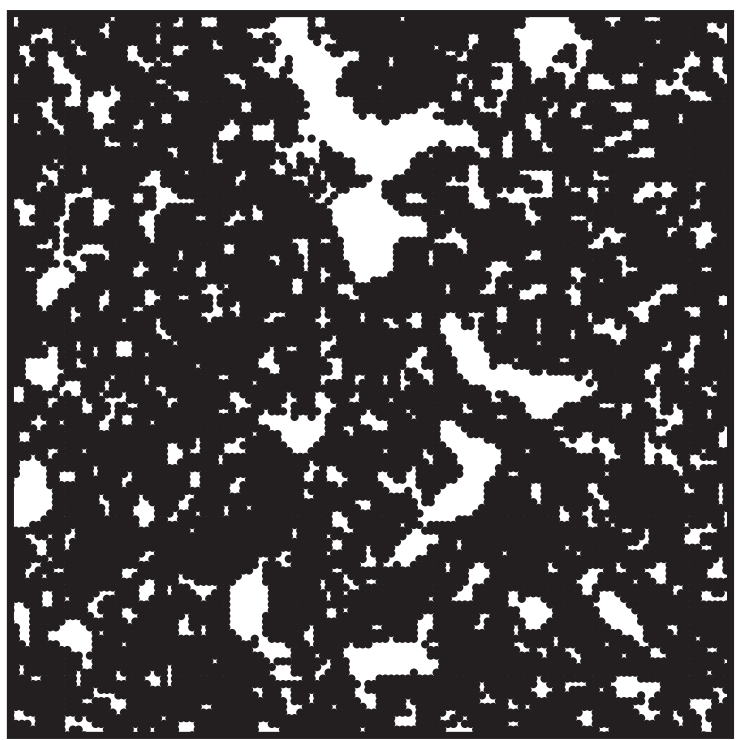

Fig. 7: Horizontal cross sections of (a) Berea Sandstone imaged with X-ray tomography and (b) virtual sample generated using the proposed method to have similar characteristics as (a). The Minkowski functionals and permeabilities of both samples are provided in Table 5 .

\section{Summary and Remarks}

We have provided a description and computational investigation of an efficient method to stochastically generate realistic pore-spaces with desired geometric and topological properties. The method consists of 
generating a realization of a correlated Gaussian field, $T(\mathbf{x})$, by convolving an underlying field of independent uniformly distributed random variables with a deterministic kernel. Then a point $\mathbf{x}$ is assigned to the pore space if $T(\mathbf{x})$ is below a threshold. Otherwise $\mathbf{x}$ is assigned to the solid matrix. A formal description of the method and statistical properties of the topographies and pore spaces are provided (Sec. 2) along with a computational investigation of how the generation parameters influence the resulting pore space (Sec. 3). In particular, the intrinsic length scales of the kernel and the applied level threshold can be used to generate pore spaces with desired values of the first three Minkowski functionals. Extensions of the basic method including creating pore spaces with multiple pore structures and preferential direction of flow are provided (Sec. 4). To illustrate how the method can be used to create samples with desired Minkowski functionals, we create a realization based on the Minkowski functionals of an imaged sample of Berea sandstone (Sec. 5).

The method is highly parallel and can produce large numbers of explicit pore spaces quickly and at low cost. Nonetheless, there are a few issues that should be considered during the generation of each pore space. The method can produce pore spaces which include suspended solids. In cases where they are unphysical the suspended solids should be removed. For low values of the threshold parameter $\gamma$, these suspended volumes do not exist. So long as $\gamma$ is below 0.55 , less than one-one hundredth of a percent of the solid volume is suspended, based on the simulations performed in Section 3. Furthermore, comparisons between flow simulations performed through pore spaces where these suspended solids were removed and the same medium where the solids were not removed, showed a negligible difference in the overall flow (details not reported here). Once $\gamma$ is sufficiently large, greater than 0.55 , a more significant percentage of the solids in the domain are suspended. This artifact of the generation method is more prevalent in narrow kernels due to the smaller correlations in the topography. These values of $\gamma$ and $\lambda$ can produce structures that can be used to simulate flow through a fluidized bed at short time scales, i.e. times so small that grains are not moved by the flow.

The proposed method does not naturally generate pore spaces that are composed of nearly solid rock with secondary channels arising from external mechanical or thermal stresses [56]. In these cases flow often corresponds to flow through a collection of discrete, sparsely connected pipes. Therefore, caution should be used when generating pore spaces with these low porosities as the virtual pore spaces might not accurately represent realistic media even though certain physical attributes are the same. However, varying the choice of kernel will naturally produce different topographies, which in turn will create different pore structures. Furthermore, using a correlated field of random variables one can create a non-Gaussian topography and create different pore structures that might mimic other natural porous media. Investigation into how these two variants of the method produce different porous media warrants further investigation.

The methodology can also be modified to generate continuum scale permeability fields. Instead of applying the level threshold to the correlated topography, it can be mapped to a desired range of permeabilities. This field can be used as the permeability field in continuum scale simulations of flow and transport.

\section{Acknowledgments}

We thank M. Zhang for providing the sample of Berea sandstone, J.M. Hyman, A. Guadagnini and C.M. Newman for several insightful discussions and encouragement, and B. Berman for helping with image processing. We gratefully acknowledge the support of the U.S. Department of Energy through the LANL/LDRD Program for this work.

\section{References}

[1] P. K. Smolarkiewicz, C. L. Winter, Pores resolving simulation of Darcy flows, J. Comput. Phys. 229 (9) (2010) $3121-3133$.

[2] M. Matyka, A. Khalili, Z. Koza, Tortuosity-porosity relation in porous media flow, Phys. Rev. E 78 (2) (2008) 026306.

[3] J. D. Hyman, P. K. Smolarkiewicz, C. Winter, Heterogeneities of flow in stochastically generated porous media, Phys. Rev. E 86 (2012) 056701, doi:10.1103/PhysRevE.86.056701.

[4] P. Mostaghimi, M. Blunt, B. Bijeljic, Computations of Absolute Permeability on Micro-CT Images, Math. Geosci. 45 (2013) 103-125

[5] J. D. Hyman, P. K. Smolarkiewicz, C. L. Winter, Pedotransfer Functions for Permeability: A Computational Study at Pore Scales, Water Resour. Res. 49, doi:10.1002/wrcr.20170. 
[6] J. D. Hyman, C. L. Winter, Hyperbolic regions in flows through three-dimensional pore structures, Phys. Rev. E 88 (2013) 063014, doi:10.1103/PhysRevE.88.063014.

[7] S. Ovaysi, M. Piri, Direct pore-level modeling of incompressible fluid flow in porous media, J. Comput. Phys. 229 (19) (2010) 7456 - 7476, ISSN 0021-9991.

[8] A. Q. Raeini, M. J. Blunt, B. Bijeljic, Modelling two-phase flow in porous media at the pore scale using the volume-of-fluid method, J. Comput. Phys. 231 (17) (2012) 5653 - 5668, ISSN 0021-9991.

[9] M. J. Blunt, B. Bijeljic, H. Dong, O. Gharbi, S. Iglauer, P. Mostaghimi, A. Paluszny, C. Pentland, Pore-scale imaging and modelling, Adv. Water Resour. 51 (2013) 197-216.

[10] M. Griebel, M. Klitz, Homogenization and numerical simulation of flow in geometries with textile microstructures, Multiscale Model Sim 8 (4) (2010) 1439-1460.

[11] P. Koumoutsakos, I. Pivkin, F. Milde, The Fluid Mechanics of Cancer and Its Therapy, Annu. Rev. Fluid Mech. 45 (1) (2013) 325 .

[12] S. Wang, A. Karrech, K. Regenauer-Lieb, S. Chakrabati-Bell, Digital bread crumb: Creation and application, J. Food Eng 116 (4) (2013) 852-861.

[13] J. A. Quiblier, A new three-dimensional modeling technique for studying porous media, J. Colloid Interf. Sci. 98 (1) (1984) 84-102.

[14] P. M. Adler, C. G. Jacquin, J. A. Quiblier, Flow in simulated porous media, Int J. Multiphas Flow 16 (4) (1990) $691-712$.

[15] J. Yao, P. Frykman, F. Kalaydjian, J.-F. Thovert, P. M. Adler, High-order moments of the phase function for real and reconstructed model porous media: A comparison, J. Colloid Interf. Sci. 156 (2) (1993) 478-490.

[16] F. E. Latief, B. Biswal, U. Fauzi, R. Hilfer, Continuum reconstruction of the pore scale microstructure for Fontainebleau sandstone, Physica A 389 (8) (2010) 1607-1618.

[17] K. R. Mecke, Additivity, convexity, and beyond: applications of Minkowski Functionals in statistical physics, in: Statistical Physics and Spatial Statistics, Springer, 111-184, 2000.

[18] R. Hilfer, Local Porosity Theory and Stochastic Reconstruction for Porous Media, in: Statistical Physics and Spatial Statistics, Springer, 203-241, 2000

[19] D. A. Klain, A short proof of Hadwiger's characterization theorem, Mathematika 42 (02) (1995) $329-339$.

[20] J. Dunsmuir, S. Ferguson, K. D'Amico, J. Stokes, X-ray microtomography: a new tool for the characterization of porous media, in: SPE Annual Technical Conference and Exhibition, 1991.

[21] D. Wildenschild, K. A. Culligan, B. S. B. Christensen, Application of X-ray microtomography to environmental fluid flow problems. in Developments in X-Ray Tomography, Proceedings of SPIE 5535 (2004) 432-441.

[22] D. Wildenschild, A. P. Sheppard, X-ray imaging and analysis techniques for quantifying pore-scale structure and processes in subsurface porous medium systems, Adv. Water Resour. 51 (2013) 217-246.

[23] P. Iassonov, T. Gebrenegus, M. Tuller, Segmentation of X-ray computed tomography images of porous materials: A crucial step for characterization and quantitative analysis of pore structures, Water Resour. Res. 45 (9).

[24] H. Okabe, M. Blunt, Prediction of permeability for porous media reconstructed using multiple-point statistics, Phys. Rev. E $70(6)(2004) 066135$.

[25] C. Manwart, S. Torquato, R. Hilfer, Stochastic reconstruction of sandstones, Phys. Rev. E 62 (2000) 893-899, doi: 10.1103/PhysRevE.62.893.

[26] K. R. Mecke, Integral geometry in statistical physics, Int. J. Mod. Phys. B 12 (09) (1998) 861-899.

[27] R. Hilfer, Review on scale dependent characterization of the microstructure of porous media, Trans. Porous Media 46 (2-3) (2002) 373-390.

[28] C. H. Arns, M. A. Knackstedt, K. R. Mecke, Reconstructing complex materials via effective grain shapes, Phys. Rev. Lett. $91(21)(2003) 215506$.

[29] A. Duda, Z. Koza, M. Matyka, Hydraulic tortuosity in arbitrary porous media flow, Phys. Rev. E 84 (3) (2011) 036319.

[30] A. M. Tartakovsky, P. Meakin, T. D. Scheibe, R. M. Eichler West, Simulations of reactive transport and precipitation with smoothed particle hydrodynamics, J. Comput. Phys. 222 (2) (2007) 654-672.

[31] J. L. Lebowitz, H. Saleur, Percolation in strongly correlated systems, Physica A 138 (1) (1986) 194-205.

[32] J. Bricmont, J. L. Lebowitz, C. Maes, Percolation in strongly correlated systems: the massless Gaussian field, J. Stat. Phys. 48 (5-6) (1987) 1249-1268.

33] S. Sheffield, Gaussian free fields for mathematicians, Probab Theory Rel 139 (3-4) (2007) 521-541.

[34] K. S. Alexander, S. A. Molchanov, Percolation of level sets for two-dimensional random fields with lattice symmetry, J. Stat. Phys. 77 (3) (1994) 627-643.

[35] K. S. Alexander, Percolation and minimal spanning forests in infinite graphs, Ann. Probab. (1995) 87-104.

[36] H. Cremér, Mathematical Methods of Statistics (PMS-9), vol. 9, Princeton university press, 1999.

[37] M. Siena, M. Riva, J. D. Hyman, C. L. Winter, A. Guadagnini, Relationship between pore size and velocity probability distributions in stochastically generated porous media, Phys. Rev. E 89 (2014) 003000, doi:10.1103/PhysRevE.00.003000.

[38] C. H. Arns, M. A. Knackstedt, K. R. Mecke, Boolean reconstructions of complex materials: Integral geometric approach, Phys. Rev. E 80 (2009) 051303, doi:10.1103/PhysRevE.80.051303.

[39] J. Bear, Dynamics of fluids in porous media, Dover publications, 1988.

[40] S. Torquato, B. Lu, Chord-length distribution function for two-phase random media, Phys. Rev. E 47 (4) (1993) 2950.

[41] H. Dong, M. J. Blunt, Pore-network extraction from micro-computerized-tomography images, Phys. Rev. E 80 (3) (2009) 036307.

[42] J. M. Prusa, P. K. Smolarkiewicz, A. A. Wyszogrodzki, EULAG, a computational model for multiscale flows, Comput. Fluids 37 (9) (2008) 1193-1207.

[43] E. Fadlun, R. Verzicco, P. Orlandi, J. Mohd-Yusof, Combined immersed-boundary finite-difference methods for three- 
dimensional complex flow simulations, J. Comput. Phys. 161 (1) (2000) 35-60.

[44] R. Dillon, L. Fauci, A microscale model of bacterial and biofilm dynamics in porous media, Biotechno Bioeng 68 (5) (2000) $536-547$.

[45] Q. Liu, O. V. Vasilyev, A Brinkman penalization method for compressible flows in complex geometries, J. Comput. Phys. 227 (2) (2007) 946-966.

[46] P. K. Smolarkiewicz, R. Sharman, J. Weil, S. G. Perry, D. Heist, G. Bowker, Building resolving large-eddy simulations and comparison with wind tunnel experiments, J. Comput. Phys. 227 (1) (2007) 633-653.

[47] S. Kang, G. Iaccarino, F. Ham, DNS of buoyancy-dominated turbulent flows on a bluff body using the immersed boundary method, J. Comput. Phys. 228 (9) (2009) 3189-3208.

[48] C. S. Peskin, Flow patterns around heart valves: a numerical method, J. Comput. Phys. 10 (2) (1972) $252-271$.

[49] C. S. Peskin, Numerical analysis of blood flow in the heart, J. Comput. Phys. 25 (3) (1977) 220-252.

[50] R. Mittal, G. Iaccarino, Immersed boundary methods, Annu. Rev. Fluid Mech. 37 (2005) 239-261.

[51] D. Goldstein, R. Handler, L. Sirovich, Modeling a no-slip flow boundary with an external force field, J. Comput. Phys. 105 (2) (1993) 354-366.

[52] A. H. Squillacote, J. Ahrens, The Paraview Guide, Kitware, 2006.

[53] M. Zhang, Personal communication, 2012.

[54] M. Sonka, V. Hlavac, R. Boyle, Image processing, analysis, and machine vision, PWS Pub. Pacific Grove, 1999.

[55] B. Bera, S. K. Mitra, D. Vick, Understanding the micro structure of Berea Sandstone by the simultaneous use of microcomputed tomography (micro-CT) and focused ion beam-scanning electron microscopy (FIB-SEM), Micron 42 (5) (2011) $412-418$.

[56] S. N. Davis, Sandstones and shales, Hydrogeology: Boulder, Colorado, Geological Society of America, The geology of North America 2 (1988) 323-332. 
